Cite this: Phys. Chem. Chem. Phys., 2013, 15, 6737

Received 27th November 2012, Accepted 14th March 2013

DOI: $10.1039 / \mathrm{c} 3 \mathrm{cp} 44236 \mathrm{e}$

www.rsc.org/pccp

\title{
Rovibrational states of $\mathrm{ClHCl}^{-}$isotopologues up to high J: a joint theoretical and spectroscopic investigation $\dagger$
}

\author{
Peter Sebald, $\ddagger^{a}$ Rainer Oswald, ${ }^{a}$ Peter Botschwina*a and Kentarou Kawaguchi ${ }^{b}$
}

\begin{abstract}
Explicitly correlated coupled cluster theory at the $\operatorname{CCSD}\left(T^{\star}\right)$-F12b level (T. B. Adler, G. Knizia, and H.-J. Werner, J. Chem. Phys., 2007, 127, 221106) and two precise spectroscopic parameters (K. Kawaguchi, J. Chem. Phys., $1988,88,4186)$ were used to construct an accurate near-equilibrium analytical potential energy function (PEF) for the highly anharmonic centrosymmetric hydrogen-bonded complex $\mathrm{ClHCl}^{-}\left(R_{\mathrm{e}}=3.1153 \AA\right.$ A $)$. From variational calculations with that PEF, a large number of rovibrational energies of different isotopologues up to high values of the rotational quantum number $J$ was obtained. Theory helped with the assignment of lines observed by IR diode laser spectroscopy in the $\nu_{1}+\nu_{3}$ combination band of ${ }^{35} \mathrm{ClH}^{35} \mathrm{Cl}^{-}$and ${ }^{37} \mathrm{ClH}^{35} \mathrm{Cl}^{-}$and enabled us to elucidate rather subtle patterns of rovibrational interactions. Furthermore, transition dipole moments were predicted and analysed as well as unusual isotopic effects.
\end{abstract}

\section{Introduction}

The hydrogen bichloride ion $\left(\mathrm{ClHCl}^{-}\right)$may be considered as a prototype of a highly anharmonic hydrogen-bonded system with a large dissociation energy. Together with the very strongly bound anion $\mathrm{FHF}^{-}$, it is rather unique due to the fact that both species and some of their isotopologues could be investigated by high-resolution diode laser infrared (IR) spectroscopy. ${ }^{1-4}$ While a larger number of bands was observed and analysed for $\mathrm{FHF}^{-}$and $\mathrm{FDF}^{-1-3}$ precise spectroscopic information on $\mathrm{ClHCl}^{-}$ isotopologues is more limited. Published line positions are restricted to the $\nu_{3}$ bands (proton stretching vibration) of ${ }^{35} \mathrm{ClH}^{35} \mathrm{Cl}^{-}$and ${ }^{37} \mathrm{ClH}^{35} \mathrm{Cl}^{-}$, which were observed by Kawaguchi in the range 692-724 $\mathrm{cm}^{-1} .4$ For the most abundant isotopologue ${ }^{35} \mathrm{ClH}^{35} \mathrm{Cl}^{-}$, the band origin was determined to be $\nu_{3}=722.8965(2) \mathrm{cm}^{-1}$. There were indications of Coriolis interaction between the $\nu_{3}$ and $\nu_{2}$ states and an approximate treatment allowed Kawaguchi to estimate the proton bending vibrational wavenumber as $\nu_{2}=792 \pm 9 \mathrm{~cm}^{-1}$. As was noted in his paper, many weaker spectral lines were also detected in the $978 \mathrm{~cm}^{-1}$ region and

\footnotetext{
${ }^{a}$ Institute of Physical Chemistry, University of Göttingen, Tammannstraße 6, D-37077 Göttingen, Germany. E-mail: pbotsch@gwdg.de; Fax: +49 551 39-3144; Tel: +49 551 39-3133

${ }^{b}$ Department of Chemistry, Okayama University, Tsushimanaka 3-1-1,

Okayama 700-8530, Japan

† Electronic supplementary information (ESI) available. See DOI: 10.1039/ c3cp44236e

† Permanent address: Madenburgstraße 14, D-76865 Insheim, Germany.
}

attributed to the combination band $\nu_{1}+\nu_{3}$, with $\nu_{1}$ denoting the heavy-atom stretching vibration, but no detailed analysis has been published so far. Likewise, theoretical work on the spectroscopic properties of this highly anharmonic species is still scarce. Almost simultaneously with the spectroscopic work, Botschwina and coworkers ${ }^{5}$ reported a two-dimensional (2D) study of the collinear potential energy surface (PES) of $\mathrm{ClHCl}^{-}$, computed by the coupled electron pair approximation (CEPA) ${ }^{6}$ and a rather flexible basis set of 121 contracted Gaussian-type orbitals. Using that PES in variational calculations with a stretch-only vibrational Hamiltonian, wavenumbers and transition dipole moments for various stretching vibrational transitions of ${ }^{35} \mathrm{ClH}^{35} \mathrm{Cl}^{-}$and ${ }^{35} \mathrm{ClD}^{35} \mathrm{Cl}^{-}$were calculated. In particular, large transition dipole moments of 1.333 and $0.822 \mathrm{D}$ were predicted for the $\nu_{3}$ fundamental and the $\nu_{1}+\nu_{3}$ combination band of ${ }^{35} \mathrm{ClH}^{35} \mathrm{Cl}^{-}$, the band origins of which were calculated to be 768 and $1031 \mathrm{~cm}^{-1}$, respectively. One year later, Ikuta et al. ${ }^{7}$ used Møller-Plesset (MP) perturbation theory at second and fourth orders in conjunction with a moderately large basis set of $\mathrm{DZ}+(\mathrm{d}, \mathrm{p})$ quality to carry out a very approximate $1 \mathrm{D}$ and $2 \mathrm{D}$ analysis of the stretching vibrations of $\mathrm{ClHCl}^{-}$and $\mathrm{ClDCl}^{-}$. In 1991, computed rovibrational energy levels of three different isotopologues of $\mathrm{ClHCl}^{-}$were published by Špirko et al. ${ }^{8}$ The two three-dimensional (3D) PESs employed were either based on their own calculations using many-body perturbation theory at fourth order (termed PES I) or a combination with the previous stretch-only CEPA PES (termed PES II). In the calculation of rovibrational energies, an 
approximate rovibrational Hamiltonian was employed which neglects part of the vibrational angular momentum and the Coriolis-type contributions. Calculations with PES II yielded $\nu_{3}=694.4 \mathrm{~cm}^{-1}, \nu_{2}=789.1 \mathrm{~cm}^{-1}$, and $\nu_{1}+\nu_{3}=941.8 \mathrm{~cm}^{-1}$, in reasonably good agreement with the above experimental values. More recently, another 2D study was published by Del Bene and Jordan. ${ }^{9}$ At the highest level, the underlying electronic structure calculations were carried out by the standard coupled cluster method CCSD(T) ${ }^{10}$ using the Dunning-type aug-cc-pVTZ basis set $^{11-13}$ in slightly reduced form. For the wavenumbers of the stretching fundamentals, $\nu_{1}=308 \mathrm{~cm}^{-1}$ and $\nu_{3}=776 \mathrm{~cm}^{-1}$ were calculated. In 2008, an empirically corrected near-equilibrium 3D PES based on MP2 calculations with the aug-cc-pVQZ basis set ${ }^{11-13}$ was used by Sebald ${ }^{14}$ to calculate anharmonic vibrational wavenumbers and a number of spectroscopic constants for ${ }^{35} \mathrm{ClH}^{35} \mathrm{Cl}^{-},{ }^{37} \mathrm{ClH}^{35} \mathrm{Cl}^{-}$, and ${ }^{37} \mathrm{ClH}^{37} \mathrm{Cl}^{-}$. The wavenumber of the proton bending vibration was predicted to be $\nu_{2}\left({ }^{35} \mathrm{ClH}^{35} \mathrm{Cl}^{-}\right)=799.1 \mathrm{~cm}^{-1}$, well within the uncertainty of the experimental estimate. ${ }^{4}$ Particular emphasis in Sebald's paper was given to a thorough discussion of the unusual $l$-type doubling constants computed for the three isotopologues of the hydrogen bichloride ion.

The present paper is devoted to a joint spectroscopic and theoretical investigation of low-lying rovibrational states of different isotopologues of $\mathrm{ClHCl}^{-}$. On the experimental side, a detailed analysis of the combination band $\nu_{1}+\nu_{3}$ of ${ }^{35} \mathrm{ClH}^{35} \mathrm{Cl}^{-}$and ${ }^{37} \mathrm{ClH}^{35} \mathrm{Cl}^{-}$has been carried out and will be presented here. The theoretical part deals with an extensive study of various rovibrational states of $\mathrm{ClHCl}^{-}$and its isotopologues up to high values of the rotational quantum number $J$. Since current quantum-chemical calculations are not yet able to faithfully deliver rovibrational energies of such species with an accuracy of $1 \mathrm{~cm}^{-1}$ or better, we make use of precise spectroscopic information to slightly improve an already quite accurate $a b$ initio near-equilibrium potential energy surface (PES). The PES is based on calculations by explicitly correlated coupled cluster theory involving non-linear correlation factors (see, e.g., ref. 15-18 for recent reviews). That theory leads to much more rapid convergence of the correlation energies compared to the corresponding standard theory. Of particular interest to the present work is the Coriolis interaction between the proton stretching and the proton bending vibration. An approximate analysis of that interacting system $\nu_{3} / \nu_{2}$ was already made in ref. 4 , but a more extensive treatment is clearly desirable.

\section{Experimental}

The infrared diode laser spectrometer (ref. 19), equipped with a modification for discharge modulation, was used for the measurement of $\mathrm{ClHCl}^{-}$spectra as described in detail in ref. 4. Briefly, the anion was produced by an AC discharge in a mixture of $\mathrm{CHCl}_{3}(13.3 \mathrm{~Pa}), \mathrm{H}_{2}(2.66 \mathrm{~Pa})$, and $\mathrm{He}(4 \mathrm{~Pa})$. The peak-to-level current was $200 \mathrm{~mA}$ at $3.5 \mathrm{kHz}$. The observed line positions were calibrated using the $\mathrm{CD}_{3} \mathrm{~F}$ spectrum as standard. An example of observed spectra, displaying lines within the $\nu_{1}+\nu_{3}$ combination bands of ${ }^{35} \mathrm{ClH}^{35} \mathrm{Cl}^{-}$and ${ }^{37} \mathrm{ClH}^{35} \mathrm{Cl}^{-}$, is shown in

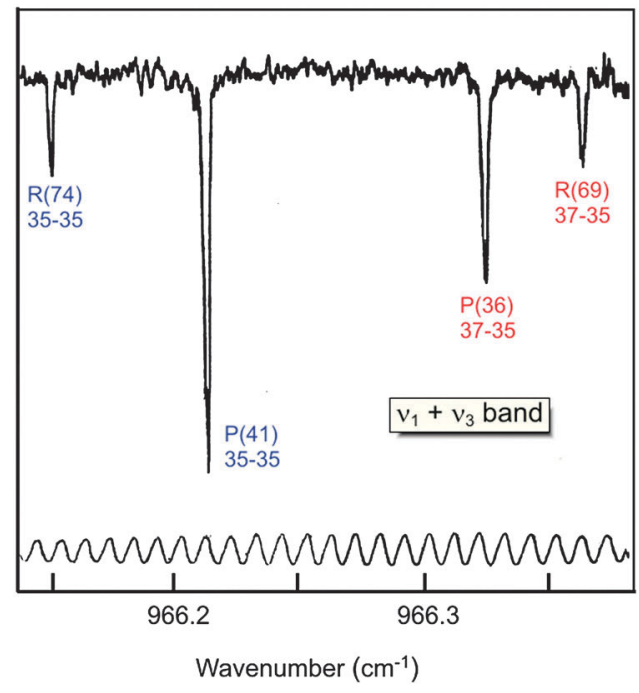

Fig. 1 Some observed lines within the $\nu_{1}+\nu_{3}$ bands of ${ }^{35} \mathrm{ClH}^{35} \mathrm{Cl}^{-}$and ${ }^{37} \mathrm{ClH}^{35} \mathrm{Cl}^{-}$.

Fig. 1, where fringes of a vacuum spaced etalon are also shown in the lower part of the figure. Over the whole wavenumber range scanned (955-990 $\mathrm{cm}^{-1}$, with many mode gaps), 45 lines could be assigned to ${ }^{35} \mathrm{ClH}^{35} \mathrm{Cl}^{-}$and 41 lines to ${ }^{37} \mathrm{ClH}^{35} \mathrm{Cl}^{-}$. The assignments are based on the previous experimental work ${ }^{4}$ and were facilitated by the theoretical study of the present paper. The ground state combination difference analysis has been performed. The observed line positions are reported in Table 1 along with the differences $\nu_{\text {obs }}-\nu_{\text {calc }}$, where the calculated wavenumbers result from a fit of the rotational energy levels by means of the formula $F_{\mathrm{v}}(J)=B_{\mathrm{v}} J(J+1)-D_{\mathrm{v}} J^{2}(J+1)^{2}$, where the collective index $\mathrm{v}$ stands for $\left(v_{1}, v_{2}^{\ell}, v_{3}\right)$, with $l$ denoting the (approximate) quantum number of the vibrational angular momentum. In contrast to the previous fits for the $\nu_{3}$ bands of the two isotopologues, ${ }^{4}$ it was not necessary to include the sextic centrifugal distortion constant $H_{\mathrm{v}}$ which would give rise to a term of power $J^{3}(J+1)^{3}$. That issue is discussed later in comparison with the theoretical results.

Spectroscopic constants for ${ }^{35} \mathrm{ClH}^{35} \mathrm{Cl}^{-}$and ${ }^{37} \mathrm{ClH}^{35} \mathrm{Cl}^{-}$are listed in Table 2, where the data for the $\nu_{3}$ state and the vibrational ground-state were taken from ref. 4 . The band origin for the main isotopic species is determined to be $\nu_{1}+\nu_{3}=$ $983.2645(2) \mathrm{cm}^{-1}$. The gas-phase value is slightly lower than the IR absorptions at 992.6 and $1001.0 \mathrm{~cm}^{-1}$ that were assigned to $\mathrm{ClHCl}^{-}$in a neon matrix-isolation study, codepositing a $\mathrm{Ne}: \mathrm{HCl}$ sample at $5 \mathrm{~K}$ with a beam of microwave excited neon atoms. ${ }^{20}$ For comparison, earlier argon-matrix experiments by Milligan and Jacox ${ }^{21}$ yielded a lower value of $956.0 \mathrm{~cm}^{-1}$, illustrating the sensitivity of the IR spectrum of $\mathrm{ClHCl}^{-}$to the environment (see ref. 20 for more details).

Changes in the rotational constants $B_{\mathrm{v}}$ between vibrational states $\nu_{1}+\nu_{3}$ and $\nu_{3}$ amount to only $0.5 \%$. On the other hand, the quartic centrifugal distortion constants $\left(D_{\mathrm{v}}\right)$ within the $\nu_{1}+\nu_{3}$ state are much closer to their ground-state counterparts than to those of the $\nu_{3}$ state. As we will see later from theoretical simulations, the $D_{\mathrm{v}}$ values of the $\nu_{3}$ states depend 
Table 1 The observed $\nu_{1}+\nu_{3}$ bands of ${ }^{35} \mathrm{ClH}^{35} \mathrm{Cl}^{-}$and ${ }^{37} \mathrm{ClH}^{35} \mathrm{Cl}^{-}\left(\mathrm{cm}^{-1}\right)$

\begin{tabular}{|c|c|c|c|c|c|}
\hline Line & $\nu_{\mathrm{obs}}$ & $\delta^{a}$ & Line & $\nu_{\mathrm{obs}}$ & $\delta^{a}$ \\
\hline \multicolumn{3}{|c|}{$\left({ }^{35} \mathrm{ClH}^{35} \mathrm{Cl}^{-}\right)$} & \multicolumn{3}{|c|}{$\left({ }^{37} \mathrm{ClH}^{35} \mathrm{Cl}^{-}\right)$} \\
\hline $\mathrm{P}(49)$ & 960.7293 & 2 & $\mathrm{P}(45)$ & 960.7698 & 1 \\
\hline $\mathrm{P}(48)$ & 961.4548 & -2 & $\mathrm{P}(44)$ & 961.4318 & 5 \\
\hline$P(45)$ & 963.5665 & 5 & $\mathrm{P}(40)$ & 963.9710 & 10 \\
\hline $\mathrm{P}(44)$ & 964.2473 & -1 & P (39) & 964.5778 & 1 \\
\hline$P(43)$ & 964.9169 & -8 & $P(36)$ & 966.3362 & -1 \\
\hline $\mathrm{P}(41)$ & 966.2250 & 1 & $\mathrm{P}(31)$ & 969.0517 & -4 \\
\hline$P(36)$ & 969.2986 & 0 & $\mathrm{P}(30)$ & 969.5628 & -2 \\
\hline$P(35)$ & 969.8799 & 0 & $\mathrm{P}(28)$ & 970.5526 & -1 \\
\hline $\mathrm{P}(34)$ & 970.4512 & 8 & $\mathrm{P}(27)$ & 971.0313 & -1 \\
\hline$P(33)$ & 971.0094 & -2 & $P(26)$ & 971.5000 & 6 \\
\hline$P(32)$ & 971.5576 & -2 & $\mathrm{P}(25)$ & 971.9566 & 0 \\
\hline$P(31)$ & 972.0944 & -5 & $\mathrm{P}(24)$ & 972.4026 & -6 \\
\hline$P(30)$ & 972.6205 & -5 & $\mathrm{P}(9)$ & 977.8151 & -3 \\
\hline$P(19)$ & 977.6772 & -3 & $\mathrm{P}(8)$ & 978.0910 & 5 \\
\hline $\mathrm{P}(18)$ & 978.0705 & -4 & $\mathrm{P}(6)$ & 978.6078 & -9 \\
\hline$P(17)$ & 978.4529 & -4 & R (69) & 966.3740 & 1 \\
\hline$P(16)$ & 978.8240 & -6 & $\mathrm{R}(43)$ & 977.6076 & 0 \\
\hline$P(15)$ & 979.1855 & 7 & $\mathrm{R}(42)$ & 977.8918 & -2 \\
\hline $\mathrm{P}(14)$ & 979.5342 & 2 & $\mathrm{R}(41)$ & 978.1648 & -7 \\
\hline$P(13)$ & 979.8729 & 7 & $\mathrm{R}(40)$ & 978.4274 & -8 \\
\hline $\mathrm{P}(11)$ & 980.5164 & 9 & R (39) & 978.6792 & -8 \\
\hline$P(10)$ & 980.8208 & 2 & $\mathrm{R}(38)$ & 978.9208 & -1 \\
\hline$P(9)$ & 981.1147 & 1 & $\mathrm{R}(37)$ & 979.1512 & 2 \\
\hline $\mathrm{R}(41)$ & 981.4453 & -8 & $\mathrm{R}(36)$ & 979.3703 & 0 \\
\hline $\mathrm{R}(42)$ & 981.1635 & -1 & $\mathrm{R}(35)$ & 979.5783 & -5 \\
\hline R (43) & 980.8699 & 1 & $\mathrm{R}(34)$ & 979.7762 & -2 \\
\hline R (44) & 980.5655 & 6 & $\mathrm{R}(33)$ & 979.9634 & 2 \\
\hline $\mathrm{R}(45)$ & 980.2484 & -3 & $\mathrm{R}(32)$ & 980.1397 & 5 \\
\hline R (46) & 979.9220 & 7 & $\mathrm{R}(31)$ & 980.3040 & -3 \\
\hline $\mathrm{R}(47)$ & 979.5825 & -1 & $\mathrm{R}(30)$ & 980.4588 & 1 \\
\hline R (48) & 979.2328 & 1 & R (29) & 980.6029 & 7 \\
\hline R (49) & 978.8712 & -4 & $\mathrm{R}(28)$ & 980.7353 & 3 \\
\hline $\mathrm{R}(50)$ & 978.4986 & -6 & $\mathrm{R}(27)$ & 980.8571 & 1 \\
\hline $\mathrm{R}(51)$ & 978.1150 & -5 & $\mathrm{R}(26)$ & 980.9687 & 5 \\
\hline R (63) & 972.6294 & -2 & $\mathrm{R}(25)$ & 981.0692 & 6 \\
\hline R (64) & 972.0978 & -9 & $\mathrm{R}(23)$ & 981.2371 & 1 \\
\hline R (65) & 971.5576 & 12 & $\mathrm{R}(22)$ & 981.3049 & -2 \\
\hline R (66) & 971.0038 & 12 & $\mathrm{R}(21)$ & 981.3622 & -3 \\
\hline R (67) & 970.4384 & 9 & R (20) & 981.4085 & -5 \\
\hline R (68) & 969.8612 & 2 & R (19) & 981.4453 & 5 \\
\hline R (69) & 969.2732 & 2 & $\mathrm{R}(18)$ & 981.4700 & 2 \\
\hline R (74) & 966.1607 & -6 & & & \\
\hline $\mathrm{R}(76)$ & 964.8357 & -5 & & & \\
\hline $\mathrm{R}(77)$ & 964.1561 & -3 & & & \\
\hline R (81) & 961.3216 & -1 & & & \\
\hline${ }^{a} \delta=($ & $\left.\nu_{\text {calc }}\right) \times 10$ & & & & \\
\hline
\end{tabular}

Table 2 Spectroscopic constants of ${ }^{35} \mathrm{ClH}^{35} \mathrm{Cl}^{-}$and ${ }^{37} \mathrm{ClH}^{35} \mathrm{Cl}^{-}\left(\mathrm{cm}^{-1}\right)^{a}$

\begin{tabular}{llll}
\hline Constant & $\begin{array}{l}\nu_{1}+\nu_{3} \text { State } \\
\text { (this work) }\end{array}$ & $\nu_{3}$ State $^{b}$ & Ground state $^{b}$ \\
\hline${ }^{35} \mathrm{ClH}^{35} \mathrm{Cl}^{-}$ & & & \\
$B_{\mathrm{v}}$ & $0.09184802(15)$ & $0.0923147(33)$ & $0.0973669(33)$ \\
$D_{\mathrm{v}} \times 10^{7}$ & $0.44717(24)$ & $0.2450(73)$ & $0.3903(66)$ \\
$H_{\mathrm{v}} \times 10^{12}$ & & $-0.97(35)$ & \\
$\nu_{0}$ & $983.2645(2)$ & $722.8965(2)$ & \\
${ }^{37} \mathrm{ClH}^{35} \mathrm{Cl}^{-}$ & & & \\
$B_{\mathrm{v}}$ & $0.08938853(21)$ & $0.0898370(37)$ & $0.0947432(32)$ \\
$D_{\mathrm{v}} \times 10^{7}$ & $0.42833(45)$ & $0.2370(82)$ & $0.3749(68)$ \\
$H_{\mathrm{v}} \times 10^{12}$ & & $-0.97(65)$ & \\
$\nu_{0}$ & $979.9062(2)$ & $722.9589(2)$ & \\
& & &
\end{tabular}

${ }^{a}$ Values in parentheses denote one standard deviation and apply to the last digits of the constants. ${ }^{b}$ Ref. 4 . very sensitively on the choice of rotational energy levels employed in their determination.

\section{Potential energy and electric dipole moment functions}

Explicitly correlated coupled cluster theory at the $\operatorname{CCSD}\left(\mathrm{T}^{*}\right)$ F12b level ${ }^{22,23}$ was used in the electronic structure calculations of the present work. The star denotes that the contributions from connected triple substitutions were scaled according to the recipe of Werner and coworkers. ${ }^{23,24}$ The atomic orbital (AO) basis set chosen for chlorine is the aug-cc-pV(5+d)Z set, ${ }^{25}$ which is combined with the aug-cc-pV5Z set for hydrogen. Both are termed AV5Z for brevity. In total, the AO basis set for $\mathrm{ClHCl}^{-}$ comprises 352 contracted Gaussian-type orbitals (cGTOs). Following the recommendations of Yousaf and Peterson, ${ }^{26}$ the additional basis sets are chosen to be AV5Z/OPTRI, V5Z/ JKFIT, ${ }^{27}$ and AV5Z/MP2FIT. ${ }^{28}$ Geminal exponents of $\beta=1.4 a_{0}{ }^{-1}$ $\left(a_{0} \approx 0.5291772 \times 10^{-10} \mathrm{~m}\right)$ were employed as suggested by Peterson et al. ${ }^{29}$ The 16 valence electrons were correlated in the $\operatorname{CCSD}\left(\mathrm{T}^{*}\right)-\mathrm{F} 12 \mathrm{~b}$ calculations which were carried out with the MOLPRO system of $a b$ initio programs. ${ }^{30}$ Computations of that sort yield results very close to the basis set limit (BSL) so that consideration of the basis set superposition error and BSL extrapolation are not considered to be necessary.

According to the previous spectroscopic ${ }^{4}$ and theoretical work, ${ }^{5,7-9} \mathrm{ClHCl}^{-}$has a centrosymmetric linear equilibrium structure that is characterized by the single geometric parameter $r_{\mathrm{e}}$ (Cl-H equilibrium distance). Using $\operatorname{CCSD}\left(\mathrm{T}^{*}\right)$-F12b in conjunction with the AV5Z basis set, $r_{\mathrm{e}}$ was calculated to be $1.55940 \AA$, with a total energy of $V_{\mathrm{e}}=-920.2541389 E_{\mathrm{h}}\left(E_{\mathrm{h}} \approx\right.$ $\left.4.35974394 \times 10^{-18} \mathrm{~J}\right)$. Analogous calculations for $\mathrm{HCl}\left(\right.$ at $r_{\mathrm{e}}=$ $1.27622 \AA)$ and $\mathrm{Cl}^{-}$yield total energies of $-460.3766790 E_{\mathrm{h}}$ and $-459.8402219 E_{\mathrm{h}}$, respectively, so that the equilibrium dissociation energy $D_{\mathrm{e}}$ of $\mathrm{ClHCl}^{-}$for fragmentation into $\mathrm{Cl}^{-}+$ $\mathrm{HCl}$ amounts to $0.0372380 E_{\mathrm{h}}$ or $8172.8 \mathrm{~cm}^{-1}$. We have also carried out standard $\operatorname{CCSD}(\mathrm{T})$ calculations with the large aug-cc-pCV6Z basis set which comprises 775 cGTOs in the case of $\mathrm{ClHCl}^{-}\left(r_{\mathrm{e}}=\right.$ $\left.1.55691 \AA, V_{\mathrm{e}}=-920.9609051 E_{\mathrm{h}}\right)$. Except for the 1s electrons of the chlorine atoms, all electrons were correlated in those calculations. At this level of theory, $D_{\mathrm{e}}$ is obtained to be $8186.3 \mathrm{~cm}^{-1}$, only $0.17 \%$ higher than the above $\operatorname{CCSD}\left(\mathrm{T}^{*}\right)$-F12b value.

In order to construct a near-equilibrium PES for $\mathrm{ClHCl}^{-}, 1057$ symmetry-unique energy points were calculated by $\operatorname{CCSD}\left(\mathrm{T}^{*}\right)-\mathrm{F} 12 \mathrm{~b} / \mathrm{AV} 5 \mathrm{Z}$, with relative energies of up to $10000 \mathrm{~cm}^{-1}$ above equilibrium. Thereby, the energetic range of interest to the spectroscopic part of the present work, extending up to vibrational state $\left(4,0^{0}, 1\right)$ at $c a .3000 \mathrm{~cm}^{-1}$, is recovered well. After some tests we decided to set up an analytical potential energy function (PEF) as a polynomial expansion in symmetry coordinates which are equivalent to those employed by Špirko et al.: ${ }^{8}$

$$
V-V_{\mathrm{e}}=\sum_{i j k} \mathrm{C}_{i j k} S_{1}^{i} S_{2}^{j} S_{3}^{k} \quad j \text { and } k: \text { even }
$$


In eqn (1), the symmetry coordinates $S_{1}-S_{3}$ are defined as follows:

$$
\begin{gathered}
S_{1}=\frac{1}{\sqrt{2}}\left(R-2 r_{\mathrm{e}}\right) \\
S_{2}=\frac{2}{r_{\mathrm{e}}} x \\
S_{3}=\sqrt{2} z
\end{gathered}
$$

In eqn ( $2 \mathrm{a}-\mathrm{c}), R$ is the instantaneous distance between the two chlorine nuclei, $z$ describes the elongation of the proton out of its equilibrium position along the $\mathrm{Cl}-\mathrm{Cl}$ axis, and $x$ measures the perpendicular distance of the proton from that axis. Weighted least-squares fitting was employed to determine the linear parameters $\mathrm{C}_{i j k}$, with the weight per energy point $E_{i}$ chosen to be $w_{i}=1 /\left(E_{i}+750 \mathrm{~cm}^{-1}\right) .{ }^{2}$ A fit with 361 parameters led to a standard deviation of $1.09 \mathrm{~cm}^{-1} .153$ of those coefficients turned out to be smaller than $10^{-6}$ a.u. and were neglected. The neglect has very little influence on the energies in the region of interest. Using the reduced fit (208 coefficients), all ab initio energy points up to $3000 \mathrm{~cm}^{-1}$ above the energy minimum are reproduced with a standard deviation of $0.6 \mathrm{~cm}^{-1}$.

The ab initio PEF was empirically improved by making use of two precise experimental data for ${ }^{35} \mathrm{ClH}^{35} \mathrm{Cl}^{-}, 4$ the ground-state rotational constant $\left(B_{000}\right)$ and the band origin of the protonstretching vibration $\left(\nu_{3}\right)$. Two parameters, $r_{\mathrm{e}}$ and a scaling factor for symmetry coordinate $S_{3}$, were adjusted to those experimental data by means of rovibrational calculations up to $J=10$. The experimental values were reproduced by changing the equilibrium bond length to $r_{\mathrm{e}}=1.55766 \AA$ and scaling $S_{3}$ with a factor of 1.00072727 . The small change in $r_{\mathrm{e}}(0.00174 \AA$ or $0.1 \%$ ) and the tiny deviation of the scaling factor from unity are indications that the uncorrected PEF is already of high quality. Since the definition of $S_{2}$ depends on $r_{\mathrm{e}}$, all relevant coefficients $\mathrm{C}_{i j k}$ were scaled with a factor of $(1.55766 / 1.55940)^{j}$. The 208 linear parameters of the resulting empirically corrected PEF are provided in the ESI $\dagger$ (Table S1), along with some further details. Some impression of the near-equilibrium PES of $\mathrm{ClHCl}^{-}$is given by Fig. 2, which shows contour lines of relative energies in the $z x$-plane for four different values of the heavy-atom distance $R$. The characteristic development of a double-minimum potential upon increase in $R$ is clearly obvious as is the strong coupling between coordinates $z$ and $x$.

The calculation of transition dipole moments and IR intensities requires the knowledge of the variation of the electric dipole moment vector $\overrightarrow{\boldsymbol{\mu}}$ with the nuclear coordinates. In the vicinity of the equilibrium structure, the electric dipole moment function (EDMF) of $\mathrm{ClHCl}^{-}$is much simpler than its $\mathrm{PEF}$, and we have decided to represent it analytically in the same way as in our previous applications to $\mathrm{AH}_{2}$ molecules. ${ }^{31-33}$ Individual values for $\overrightarrow{\boldsymbol{\mu}}$ were calculated by the finite field technique (field strength: 0.0003 a.u.) at 175 symmetry-unique nuclear configurations and were then transformed to the molecular Eckart frame. Subsequently, the resulting values of

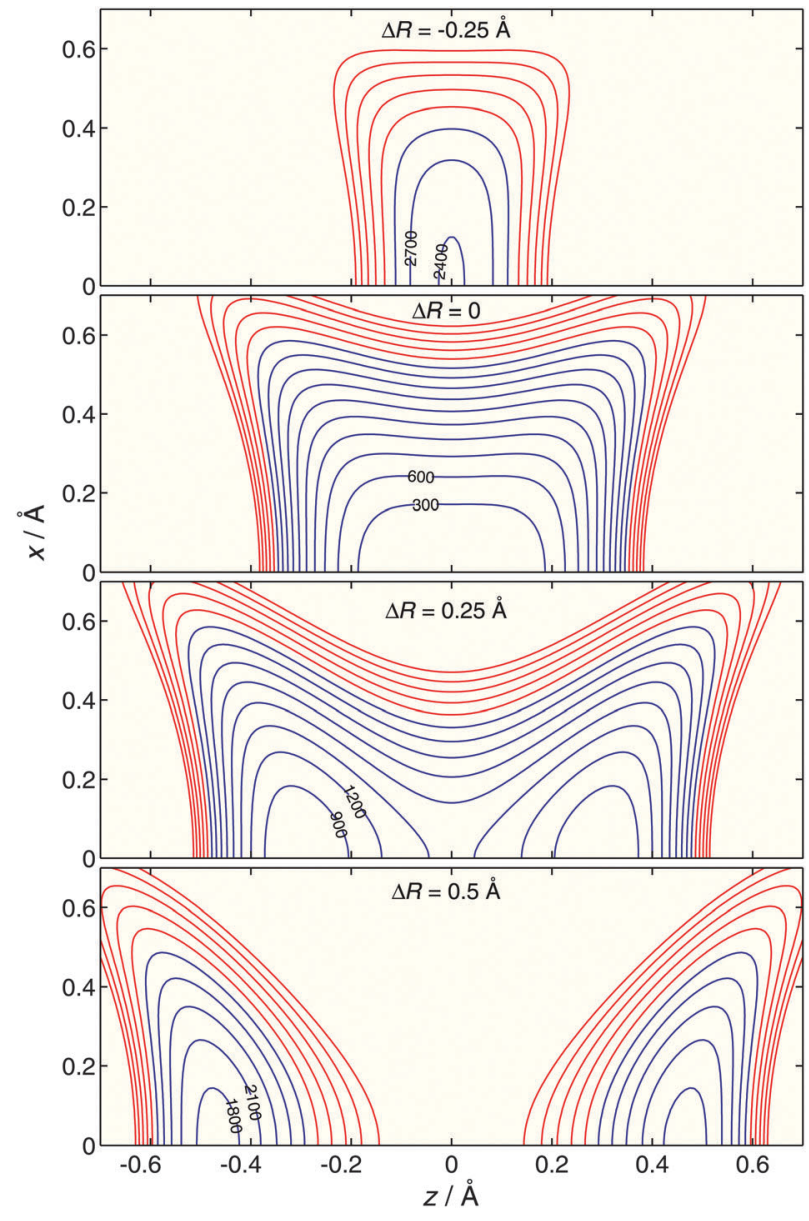

Fig. 2 Two-dimensional contour-plots of the potential energy function for $\mathrm{ClHCl}^{-}$. Contour lines in intervals of $300 \mathrm{~cm}^{-1}$ with zero at the absolute energy minimum. Contour lines above $3000 \mathrm{~cm}^{-1}$ are drawn in red (see the text).

the parallel and perpendicular components $\left(\mu^{\prime \prime}\right.$ and $\left.\mu^{\perp}\right)$ where fitted to the expression

$$
\mu^{\alpha}=\sum_{i j k} D_{i j k}^{\alpha} \tilde{S}_{1}^{i} \tilde{S}_{3}^{j} \theta^{k} \quad \alpha: \text { parallel or perpendicular }
$$

In eqn (3), the coordinates $\tilde{S}_{1}$ and $\tilde{S}_{3}$ are defined as follows:

$$
\tilde{S}_{1}=\frac{1}{\sqrt{2}}\left(r_{1}+r_{2}-2 r_{\mathrm{e}}\right) \text { and } \tilde{S}_{3}=\frac{1}{\sqrt{2}}\left(r_{1}-r_{2}\right)
$$

Here, $r_{1}$ and $r_{2}$ are the proton-chlorine distances. For a linear arrangement of the nuclei, the coordinate $\tilde{S}_{1}$ is identical to $S_{1}$ of eqn (2a) and the coordinate $\tilde{S}_{3}$ to $S_{3}$ of eqn (2c). The coordinate $\theta$ measures the deviation of the $\mathrm{ClHCl}$ angle from linearity. As is shown in Fig. 3, the parallel component of the electric dipole moment at $\theta=0^{\circ}$ varies strongly only with the proton stretching coordinate $\tilde{S}_{3}$ and that variation is almost linear for $\tilde{S}_{3} \leq 0.5 a_{0}$. On the other hand, $\mu_{\perp}$ changes only slightly with the angle $\theta$; even for a large value of $\theta=60^{\circ}$, only a small value of $\mu_{\perp} \approx$ $0.1 \mathrm{ea}_{0}$ is computed. 


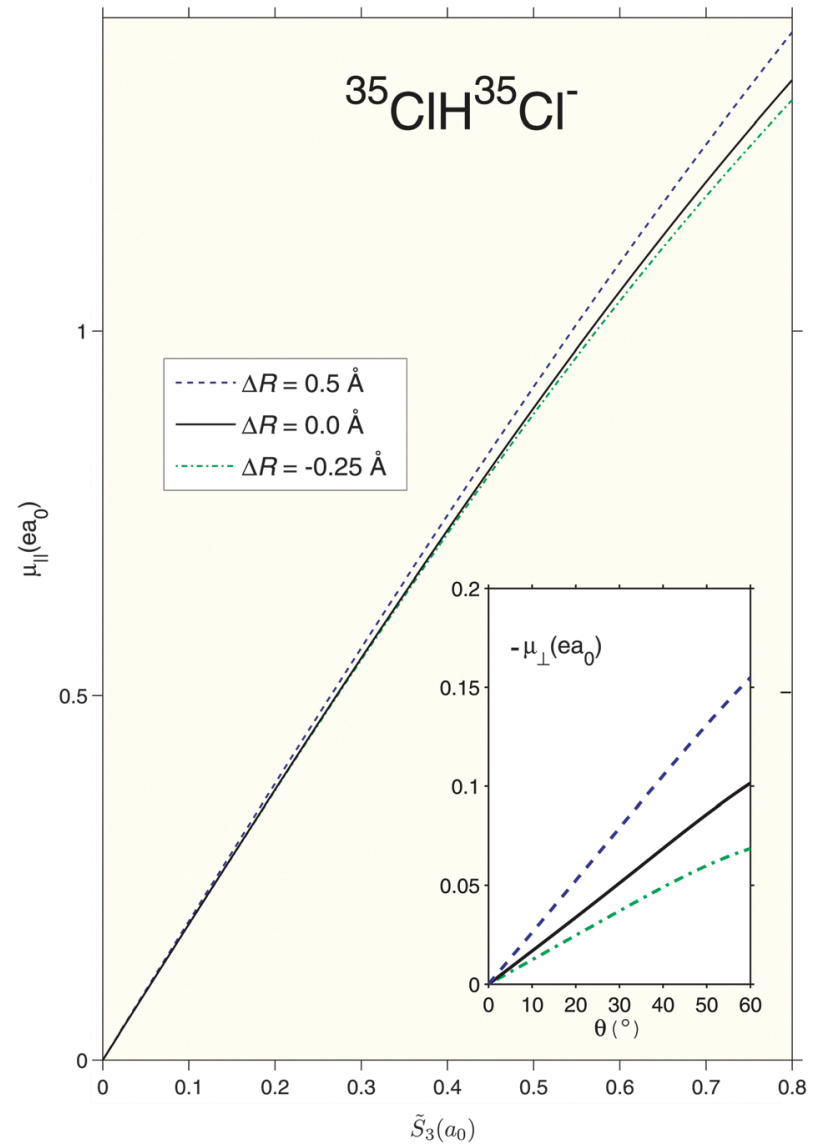

Fig. 3 Variation of the electric dipole moment of ${ }^{35} \mathrm{ClH}^{35} \mathrm{Cl}^{-}$with the antisymmetric stretching coordinate $\tilde{S}_{3}$ and the angle $\theta$ (deviation from linearity).

\section{Rovibrational states and their interaction}

\subsection{Details of calculations}

The calculation of rovibrational energies and wave functions for $\mathrm{ClHCl}^{-}$isotopologues is based on Watson's isomorphic Hamiltonian for linear molecules, which is represented in normal coordinates $Q_{k}$ and the corresponding conjugate momenta $P_{k}{ }^{34}$ For a linear triatomic species with four vibrational degrees of freedom it may be written as follows:

$$
\begin{aligned}
\hat{H}= & \frac{1}{2} \sum_{k=1}^{4} P_{k}^{2}+\frac{1}{2} \mu\left(\pi_{x}^{2}+\pi_{y}^{2}\right)+\frac{1}{2} \mu\left(\Pi_{x}^{\prime 2}+\Pi_{y}^{\prime 2}\right) \\
& -\mu\left(\Pi_{x}^{\prime} \pi_{x}+\Pi_{y}^{\prime} \pi_{y}\right)+V
\end{aligned}
$$

The first two terms of $\hat{H}$ constitute the vibrational kinetic energy part of the Hamiltonian, termed $\hat{H}_{\text {vib }}$, the third the rotational contribution $\left(\hat{H}_{\text {rot }}\right)$ and the fourth the Coriolis part $\left(\hat{H}_{\text {cor }}\right)$. As usual, the potential energy is denoted by $V$. For $\mathrm{ClHCl}^{-}$isotopologues, the second term is rather insignificant. For the low-lying vibrational states of interest to the present work its maximum contribution to the vibrational term energies does not exceed $0.3 \mathrm{~cm}^{-1}$.

The matrix elements of $\hat{H}$ were calculated in a basis of products of symmetrized harmonic oscillator/rigid rotor
(HO/RR) functions. Specifically, one-dimensional HO functions were used for the stretching vibrations $\left(\nu_{1}\right.$ and $\left.\nu_{3}\right)$ whereas twodimensional HO functions (TDHOs) with quantum numbers $\mathrm{v}_{2}$ and $l$ were employed for the bending vibration $\left(\nu_{2}\right)$. Integration over normal coordinates was performed by Gauss-Hermite and Gauss-Laguerre integration as suggested by Whitehead and Handy, ${ }^{35}$ while integration over the Euler angles was performed analytically. ${ }^{36}$ For the symmetric isotopologue ${ }^{35} \mathrm{ClH}^{35} \mathrm{Cl}^{-}$, the vibrational basis set (for $J=0$ ) was carefully selected and comprises 1136 HO products. A comparable vibrational basis of $2272 \mathrm{HO}$ products was chosen for the asymmetric isotopologues. Although the present work deals with rovibrational states up to $J=130$, we may well restrict the choice of TDHOs to relatively low $l$ values. In our calculations for $\mathrm{ClHCl}^{-}$ isotopologues, the maximum value is chosen to be $l_{\max }=15$. Since $\mathrm{v}_{2} \geq l$ and the harmonic wavenumber $\omega_{2}\left({ }^{35} \mathrm{ClH}^{35} \mathrm{Cl}^{-}\right)$is as high as $828 \mathrm{~cm}^{-1}$, the diagonal matrix element $\left\langle 0,15^{15}\right.$, $\left.0|\hat{H}| 0,15^{15}, 0\right\rangle$ has an energy of almost $12000 \mathrm{~cm}^{-1}$ above the vibrational ground state. The influence of such a basis function on the low-lying vibrational states of interest here (stretch-only vibrational states and states with $\mathrm{v}_{2}=1$ ) is vanishingly small and basis functions with higher $l$ values may thus be neglected. With the given choice of $l_{\text {max }}$, the maximum number of $\mathrm{HO} / \mathrm{RR}$ basis functions is 18176 for symmetric and 36352 for asymmetric isotopologues.

Squared transition dipole moments $\mu_{\mathrm{if}}^{2}$ between initial rovibrational state $\mathrm{i}$ and final state $\mathrm{f}$ were calculated from the rovibrational wavefunctions and the EDMF of Table S2 (ESI $\dagger$ ), closely following the detailed description of ref. 37 . They are conveniently written as the product of three factors:

$$
\mu_{\mathrm{if}}^{2} \approx F_{\mathrm{HL}} F_{\mathrm{HW}} \mu_{\mathrm{vv}^{\prime}}^{2}
$$

In eqn (6), $\mu_{\mathrm{vv}^{\prime}}$ is the transition dipole moment of the pure vibrational transition, $F_{\mathrm{HL}}$ the Hönl-London factor, ${ }^{36}$ and $F_{\mathrm{HW}}$ the Herman-Wallis factor. ${ }^{38}$ For $F_{\mathrm{HW}}$ an expression of the form $\left(1+\mathrm{A}_{1} m\right)^{2}$ was used with $m=-J$ and $m=J+1$ for P-branch and R-branch transitions, respectively. ${ }^{39}$ Throughout this paper, transition dipole moments are quoted in convenient units of debye $\left(1 \mathrm{D} \approx 3.33564 \times 10^{-30} \mathrm{C} \mathrm{m}\right)$.

\subsection{Vibrational ground state and pure stretching vibrational states of $\mathbf{H}$ isotopologues}

We start with those vibrational states for which direct experimental information has become available through diode laser IR absorption spectroscopy. ${ }^{4}$ These are the ground vibrational state $\left(0,0^{0}, 0\right)$ and the stretching vibrational states $\left(0,0^{0}, 1\right)$ and $\left(1,0^{0}, 1\right)$. Calculated rovibrational term energies for the isotopologues ${ }^{35} \mathrm{ClH}^{35} \mathrm{Cl}^{-},{ }^{37} \mathrm{ClH}^{35} \mathrm{Cl}^{-}$, and ${ }^{37} \mathrm{ClH}^{37} \mathrm{Cl}^{-}$up to $J_{\text {max }}=110$ are listed in Table S3, ESI. $\uparrow$ The zero-point energies of the three species are 1347.72, 1345.36, and $1342.95 \mathrm{~cm}^{-1}$. Effective spectroscopic constants were calculated within each vibrational state $\{\mathrm{v}\}$ by a least-squares fit with the common power expansion in $J(J+1)$, producing $B_{\mathrm{v}}, D_{\mathrm{v}}$, (and $H_{\mathrm{v}}$ ) along with the band origins $\left(\nu_{3}\right.$ and $\left.\nu_{1}+\nu_{3}\right)$. Results of a variety of fits, differing in $J_{\max }$ and the choice of the fitting parameters, are 
listed in Table 3 for ${ }^{35} \mathrm{ClH}^{35} \mathrm{Cl}^{-}$and ${ }^{37} \mathrm{ClH}^{35} \mathrm{Cl}^{-}$, experimental results from Table 2 being included as well.

For the vibrational ground state, fits including the parameters $B_{000}$ and $D_{000}$ and with choice of $J_{\max }=10,30,50$, or 70 yield the rotational constant stable within $10^{-6} \mathrm{~cm}^{-1}$ and lead to changes in $D_{000}$ of less than $10^{-10} \mathrm{~cm}^{-1}$. Inclusion of the $H_{000}$ term at $J_{\max }=90$ and 110 leads to tiny values for this constant and produces insignificant changes in $B_{000}$ and $D_{000}$. Owing to the chosen PEF adjustment, theoretical and experimental $B_{000}$ values for ${ }^{35} \mathrm{ClH}^{35} \mathrm{Cl}^{-}$are identical and the difference in $B_{000}$ $\left({ }^{37} \mathrm{ClH}^{35} \mathrm{Cl}^{-}\right)$is within experimental uncertainty. The same holds for the quartic centrifugal distortion constant $D_{000}$ of both isotopologues. The situation for the first excited state of the proton stretching vibration $\left(0,0^{\circ}, 1\right)$ is rather different. In this case, $D_{001}$ depends strongly on $J_{\max }$ when the fit is restricted to $B_{001}$ and $D_{001}$ terms, varying between $0.2434 \times$ $10^{-7} \mathrm{~cm}^{-1}$ for $J_{\max }=10$ and $0.3842 \times 10^{-7} \mathrm{~cm}^{-1}$ for $J_{\max }=110$ (for ${ }^{35} \mathrm{ClH}^{35} \mathrm{Cl}^{-}$). As was recognized by Kawaguchi, ${ }^{4}$ Coriolis interaction with the higher-lying bending vibrational state $\left(0,1^{1}, 0\right)$ may be responsible for that behavior. We will address this issue in detail in Section 4.4. Despite the high sensitivity of
$D_{001}$ and $H_{001}$, agreement between theory and experiment is quite good, provided that exactly the same rovibrational transitions are included in the fits. The theoretical values for the $\left(0,0^{0}, 1\right)$ states of ${ }^{35} \mathrm{ClH}^{35} \mathrm{Cl}^{-}$and ${ }^{37} \mathrm{ClH}^{35} \mathrm{Cl}^{-}$, determined as described in footnote $\mathrm{d}$ of Table 3 , agree with experiment within $9 \%$ for $D_{001}$ and within $16 \%$ for $H_{001}$, i.e., the latter well within the large standard deviations of the experimental values.

Somewhat surprisingly, fits of rotational levels within the $\left(1,0^{\circ}, 1\right)$ state without inclusion of the $H_{101}$ term show excellent performance up to $J_{\max }=110$, leading to very stable values for both the rotational constant and the quartic centrifugal distortion constant of both isotopologues. As we will see in Section 4.4, theory will provide an explanation for that behavior which is in line with the available spectroscopic data (cf. Section 2). Using the same rovibrational transitions as in the analysis of the observed lines, values for $B_{101}$ and $D_{101}$ are obtained that differ from the experimental values by only $-0.05 \%$ and less than $2 \%$, respectively.

The band origin of the $\nu_{3}$ band of ${ }^{37} \mathrm{ClH}^{35} \mathrm{Cl}^{-}$is calculated to be $722.96 \mathrm{~cm}^{-1}$, larger than the corresponding value of the most abundant isotopologue by $0.060 \mathrm{~cm}^{-1}\left(\exp . .^{4} 0.062 \mathrm{~cm}^{-1}\right)$.

Table 3 Effective spectroscopic constants (in $\mathrm{cm}^{-1}$ ) of ${ }^{35} \mathrm{ClH}^{35} \mathrm{Cl}^{-}$and ${ }^{37} \mathrm{ClH}^{35} \mathrm{Cl}^{-}$

\begin{tabular}{|c|c|c|c|c|c|c|c|}
\hline \multirow[b]{2}{*}{ State } & \multirow[b]{2}{*}{$J_{\max }$} & \multicolumn{3}{|l|}{${ }^{35} \mathrm{ClH}^{35} \mathrm{Cl}^{-a}$} & \multicolumn{3}{|l|}{${ }^{37} \mathrm{ClH}^{35} \mathrm{Cl}^{-b}$} \\
\hline & & $B_{\mathrm{v}}$ & $D_{\mathrm{v}}\left(\times 10^{7}\right)$ & $H_{\mathrm{v}}\left(\times 10^{12}\right)$ & $B_{\mathrm{v}}$ & $D_{\mathrm{v}}\left(\times 10^{7}\right)$ & $H_{\mathrm{v}}\left(\times 10^{12}\right)$ \\
\hline \multirow[t]{10}{*}{$\left(0,0^{0}, 0\right)$} & 10 & 0.097367 & 0.3975 & & 0.094740 & 0.3762 & \\
\hline & 30 & 0.097367 & 0.3976 & & 0.094740 & 0.3763 & \\
\hline & 50 & 0.097367 & 0.3980 & & 0.094740 & 0.3767 & \\
\hline & 70 & 0.097368 & 0.3985 & & 0.094740 & 0.3771 & \\
\hline & 90 & 0.097368 & 0.3993 & & 0.094740 & 0.3778 & \\
\hline & & 0.097367 & 0.3974 & -0.017 & 0.094740 & 0.3761 & -0.015 \\
\hline & 110 & 0.097369 & 0.4003 & & 0.094741 & 0.3787 & \\
\hline & & 0.097367 & 0.3972 & -0.018 & 0.094740 & 0.3760 & -0.016 \\
\hline & $d$ & 0.097368 & 0.3984 & & 0.094742 & 0.3804 & \\
\hline & Exp. $^{c}$ & $0.097367(3)$ & $0.3903(66)$ & & $0.094743(4)$ & $0.3749(68)$ & \\
\hline \multirow[t]{12}{*}{$\left(0,0^{0}, 1\right)$} & 10 & 0.092281 & 0.2434 & & 0.089801 & 0.2365 & \\
\hline & 30 & 0.092282 & 0.2622 & & 0.089802 & 0.2533 & \\
\hline & 50 & 0.092285 & 0.2922 & & 0.089805 & 0.2804 & \\
\hline & & 0.092281 & 0.2480 & -1.248 & 0.089801 & 0.2405 & -1.124 \\
\hline & 70 & 0.092294 & 0.3257 & & 0.089812 & 0.3106 & \\
\hline & & 0.092283 & 0.2615 & -0.934 & 0.089803 & 0.2525 & -0.847 \\
\hline & 90 & 0.092307 & 0.3571 & & 0.089825 & 0.3393 & \\
\hline & & 0.092286 & 0.2812 & -0.672 & 0.089806 & 0.2700 & -0.614 \\
\hline & 110 & 0.092326 & 0.3842 & & 0.089842 & 0.3642 & \\
\hline & & 0.092293 & 0.3038 & -0.478 & 0.089811 & 0.2904 & -0.439 \\
\hline & $d$ & 0.092283 & 0.2625 & -0.91 & 0.089805 & 0.2570 & -0.82 \\
\hline & Exp. $^{c}$ & $0.092315(3)$ & $0.2450(73)$ & $-0.97(35)$ & $0.089837(4)$ & $0.2370(82)$ & $-0.97(65)$ \\
\hline \multirow[t]{11}{*}{$\left(1,0^{0}, 1\right)$} & 10 & 0.091805 & 0.4507 & & 0.089342 & 0.4268 & \\
\hline & 30 & 0.091805 & 0.4518 & & 0.089342 & 0.4279 & \\
\hline & 50 & 0.091805 & 0.4537 & & 0.089343 & 0.4294 & \\
\hline & 70 & 0.091805 & 0.4556 & & 0.089343 & 0.4310 & \\
\hline & & 0.091805 & 0.4522 & -0.049 & 0.089343 & 0.4282 & -0.040 \\
\hline & 90 & 0.091806 & 0.4564 & & 0.089343 & 0.4315 & \\
\hline & & 0.091805 & 0.4551 & -0.011 & 0.089343 & 0.4309 & -0.005 \\
\hline & 110 & 0.091804 & 0.4542 & & 0.089342 & 0.4293 & \\
\hline & & 0.091807 & 0.4621 & 0.047 & 0.089345 & 0.4372 & 0.047 \\
\hline & $e$ & 0.091806 & 0.4558 & & 0.0893453 & 0.4337 & \\
\hline & Exp. $^{c}$ & $0.091848(2)$ & $0.4472(3)$ & & $0.0893885(2)$ & $0.4283(5)$ & \\
\hline
\end{tabular}

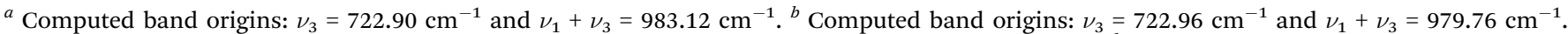

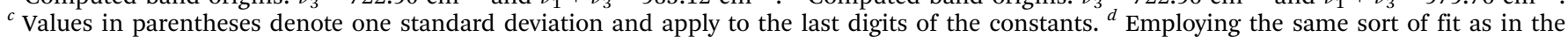
previous experimental paper (ref. 4). ${ }^{e}$ Employing the same sort of fit as in the present experimental part (see Section 2 ). 
As was mentioned earlier, ${ }^{5}$ such an increase cannot occur within the harmonic approximation and must be due to anharmonicity. The unusual isotope effect is twice as large for ${ }^{37} \mathrm{ClH}^{37} \mathrm{Cl}^{-}$. The still unknown experimental value for the band origin of that isotopologue is thus predicted to be $\nu_{3}\left({ }^{37} \mathrm{ClH}^{37} \mathrm{Cl}^{-}\right)=$ $723.02 \mathrm{~cm}^{-1}$. The corresponding rotational and centrifugal distortion constants are calculated to be $B_{001}=0.08732 \mathrm{~cm}^{-1}, D_{001}=$ $0.243 \times 10^{-7} \mathrm{~cm}^{-1}$, and $H_{001}=-0.77 \times 10^{-12} \mathrm{~cm}^{-1}$, obtained through a least-squares fit to rovibrational energies up to $J_{\max }=$ 70. The ground-state values of ${ }^{37} \mathrm{ClH}^{37} \mathrm{Cl}^{-}$are predicted to be $B_{000}=0.09211 \mathrm{~cm}^{-1}$ and $D_{000}=0.356 \times 10^{-7} \mathrm{~cm}^{-1}$. For the combination vibrational state $\left(1,0^{0}, 1\right)$ we obtain $B_{101}=$ $0.08688 \mathrm{~cm}^{-1}$ and $D_{101}=0.404 \times 10^{-7} \mathrm{~cm}^{-1}$, again with very stable results for fits with $J_{\max }$ up to 110 .

Compared to experiment, the calculated band origins of the $\nu_{1}+\nu_{3}$ bands of ${ }^{35} \mathrm{ClH}^{35} \mathrm{Cl}^{-}$and ${ }^{37} \mathrm{ClH}^{35} \mathrm{Cl}^{-}$are underestimated by 0.14 and $0.15 \mathrm{~cm}^{-1}$, respectively. For ${ }^{37} \mathrm{ClH}^{37} \mathrm{Cl}^{-}$, we calculate $\nu_{1}+\nu_{3}=976.36 \mathrm{~cm}^{-1}$ and, assuming an error of $-0.16 \mathrm{~cm}^{-1}$, we would like to make the prediction $\nu_{1}+\nu_{3}$ $\left({ }^{37} \mathrm{ClH}^{37} \mathrm{Cl}^{-}\right)=976.52 \pm 0.02 \mathrm{~cm}^{-1}$.

Predictions for the stretching vibrational states of the overtone series $n \nu_{1}(n=1-4)$ are made in Table 4 . All the four states of the three isotopologues considered appear to be essentially unperturbed up to high $J$ values and therefore three spectroscopic constants $\left(G_{\mathrm{v}}, B_{\mathrm{v}}\right.$, and $\left.D_{\mathrm{v}}\right)$ were determined by a least-squares fit to rotational levels up to $J_{\max }=30$. Regarding the small errors obtained for the $\nu_{1}+\nu_{3}$ combination tones of ${ }^{35} \mathrm{ClH}^{35} \mathrm{Cl}^{-}$and ${ }^{37} \mathrm{ClH}^{35} \mathrm{Cl}^{-}$, we are confident that the errors in the $\nu_{1}$ fundamentals will be smaller than $0.2 \mathrm{~cm}^{-1}$ and those for the overtones up to $4 \nu_{1}$ should not exceed $1 \mathrm{~cm}^{-1}$. Since the wavenumbers for the $\nu_{1}+\nu_{3}$ bands are underestimated, we may expect that the wavenumbers of the $n \nu_{1}$ series are slightly too small, as well. For ${ }^{35} \mathrm{ClH}^{35} \mathrm{Cl}^{-}$, the change in rotational constant upon excitation of the totally symmetric $\mathrm{Cl}-\mathrm{Cl}$ stretching vibration by 1-4 quanta is predicted to be $-0.59 \%,-1.31 \%,-2.26 \%$, and $-3.42 \%$. Much larger changes are calculated for the quartic centrifugal constant, which increases by $6.9 \%, 18.6 \%, 36.4 \%$, and $56.6 \%$.

\subsection{Vibrational states $\left(0,1^{1}, 0\right)$ and $\left(1,1^{1}, 0\right)$ of $H$ isotopologues}

In the previous experimental work, ${ }^{4}$ approximate information about the proton bending vibration $\nu_{2}$ of ${ }^{35} \mathrm{ClH}^{35} \mathrm{Cl}^{-}$was obtained through a two-state Coriolis interaction model involving vibrational states $\left(0,0^{0}, 1\right)$ and $\left(0,1^{1}, 0\right)$. That model is adequate for the case of Coriolis resonance, but may be less suitable for the case of weaker Coriolis interaction. In addition,

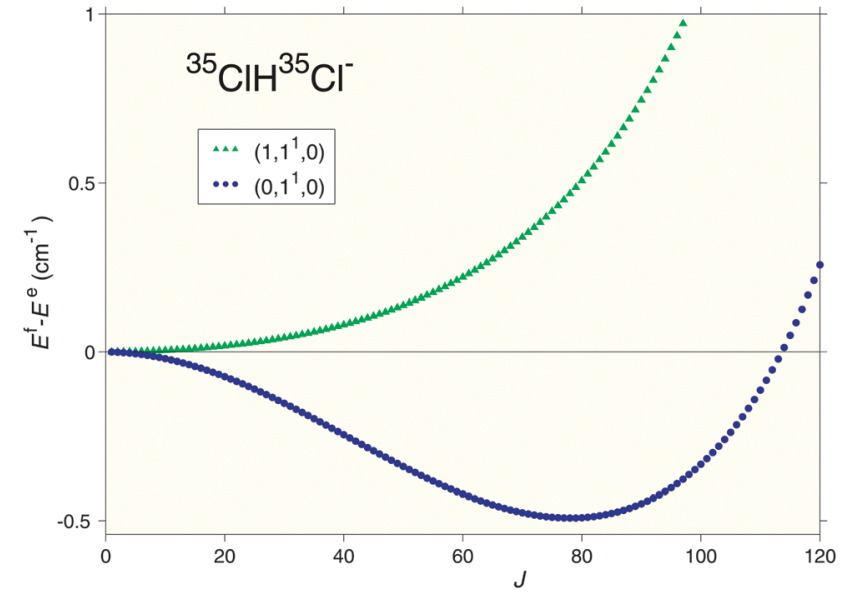

Fig. 4 Calculated splitting between $f$ and e sublevels of ${ }^{35} \mathrm{ClH}^{35} \mathrm{Cl}^{-}$in the $\left(0,1^{1}, 0\right)$ and $\left(1,1^{1}, 0\right)$ vibrational states.

a guess had to be made for the Coriolis coupling constant $\eta$ in order to arrive at an estimate of $\nu_{2}=792 \pm 9 \mathrm{~cm}^{-1}$. Published values from $a b$ initio calculations involving vibrational anharmonicity are $787.3 \mathrm{~cm}^{-1}$ (PES I) and $789.1 \mathrm{~cm}^{-1}$ (PES II) as obtained by Špirko et $a l^{8}$ and $799.1 \mathrm{~cm}^{-1}$ as computed by Sebald, ${ }^{14}$ all in agreement with Kawaguchi's estimate.

Using the complete Watson Hamiltonian ${ }^{34}$ and the PES from Section 3, rovibrational term energies have been calculated for the $l=1$ states $\left(0,1^{1}, 0\right)$ and $\left(1,1^{1}, 0\right)$ of ${ }^{35} \mathrm{ClH}^{35} \mathrm{Cl}^{-}$up to $J_{\max }=120$ (see ESI $\dagger$ Table S4). As is well known, the rotational levels within such states are split apart by Coriolis coupling and thus exhibit $l$-type doubling. ${ }^{40}$ The resulting pairs of sublevels have parity $+(-1)^{J}$ for the e sublevels and $-(-1)^{J}$ for the $\mathrm{f}$ sublevels. ${ }^{41}$ The energy differences between $\mathrm{f}$ and the corresponding e sublevels of the $\left(0,1^{1}, 0\right)$ and $\left(1,1^{1}, 0\right)$ states are plotted in Fig. 4 . As was already discussed in detail by Sebald, ${ }^{14}$ the $f$ sublevels of the $\left(0,1^{1}, 0\right)$ state are below the corresponding e sublevels up to high values of $J$, leading to negative values of the energy difference $\Delta E(J)=E^{\mathrm{f}}(J)-E^{\mathrm{e}}(J)$ in that range. According to the present calculations, the graph of the function $\Delta E(J)$ has a minimum at $J=78$ and $\Delta E(J)$ changes sign between $J=113$ and $J=114$. Such a change of $\Delta E(J)$ appears to be rather unusual and results from anharmonic interaction with other vibrational states, to be discussed in more detail in Section 4.4. On the other hand, and perhaps surprisingly, the graph of $\Delta E(J)$ for the combination vibrational state $\left(1,1^{1}, 0\right)$ shows a rather normal shape, with monotonically rising positive values over the whole range of $J$ values being considered.

Table 4 Effective spectroscopic constants of the $n \nu_{1}$ overtone series of ${ }^{35} \mathrm{ClH}^{35} \mathrm{Cl}^{-},{ }^{37} \mathrm{ClH}^{35} \mathrm{Cl}^{-}$, and ${ }^{37} \mathrm{ClH}^{37} \mathrm{Cl}^{-}$(in $\mathrm{cm}^{-1}$ )

\begin{tabular}{|c|c|c|c|c|c|c|c|c|c|}
\hline Vibrational state & \multicolumn{3}{|c|}{${ }^{35} \mathrm{ClH}^{35} \mathrm{Cl}^{-}$} & \multicolumn{3}{|c|}{${ }^{37} \mathrm{ClH}^{35} \mathrm{Cl}^{-}$} & \multicolumn{3}{|c|}{${ }^{37} \mathrm{ClH}^{37} \mathrm{Cl}^{-}$} \\
\hline$\left(1,0^{0}, 0\right)$ & 302.76 & 0.096800 & 0.4250 & 298.74 & 0.094197 & 0.4016 & 294.66 & 0.091594 & 0.3791 \\
\hline$\left(3,0^{0}, 0\right)$ & 880.84 & 0.095177 & 0.5423 & 869.73 & 0.092656 & 0.5090 & 858.45 & 0.090127 & 0.4784 \\
\hline$\left(4,0^{0}, 0\right)$ & 1150.39 & 0.094037 & 0.6226 & 1136.45 & 0.091589 & 0.6104 & 1122.28 & 0.089100 & 0.5488 \\
\hline
\end{tabular}

${ }^{a}$ Obtained from fits with $J_{\max }=30$. 
Table 5 Calculated effective spectroscopic constants (in $\mathrm{cm}^{-1}$ ) for the $\left(0,1^{1}, 0\right)$ and $\left(1,1^{1}, 0\right)$ states of ${ }^{35} \mathrm{ClH}^{35} \mathrm{Cl}^{-a}$

\begin{tabular}{lrrllll}
\hline State & $J_{\max }$ & $G_{\mathrm{v}}$ & $B_{\mathrm{v}}$ & $D_{\mathrm{v}}\left(\times 10^{7}\right)$ & $q_{\mathrm{v}}\left(\times 10^{3}\right)$ & $q_{\mathrm{D}}\left(\times 10^{7}\right)$ \\
\hline$\left(0,1^{1}, 0\right)$ & 10 & 795.75 & 0.098133 & 0.5442 & -0.184 & 0.23 \\
& 30 & 795.75 & 0.098133 & 0.5356 & -0.184 & 0.22 \\
& 50 & 795.75 & 0.098131 & 0.5224 & -0.181 & 0.19 \\
& 70 & 795.75 & 0.098128 & 0.5088 & -0.173 & 0.16 \\
& 90 & 795.75 & 0.098123 & 0.4977 & -0.163 & 0.14 \\
& 110 & 795.76 & 0.098119 & 0.4917 & -0.152 & 0.12 \\
$\left(1,1^{1}, 0\right)$ & 10 & 1090.33 & 0.097381 & 0.4942 & 0.040 & 0.05 \\
& 30 & 1090.33 & 0.097381 & 0.4949 & 0.040 & 0.05 \\
& 50 & 1090.33 & 0.097381 & 0.4967 & 0.040 & 0.06 \\
& 70 & 1090.33 & 0.097382 & 0.5003 & 0.039 & 0.06 \\
& 90 & 1090.33 & 0.097385 & 0.5071 & 0.035 & 0.07 \\
& 110 & 1090.33 & 0.097394 & 0.5205 & 0.022 & 0.09
\end{tabular}

${ }^{a}$ See eqn (7) and (8) for definition.

In order to deduce effective spectroscopic constants for the states $\left(0,1^{1}, 0\right)$ and $\left(1,1^{1}, 0\right)$, we have fitted the energies of their e and $f$ sublevels with the formulae

$$
\begin{gathered}
\frac{E^{\mathrm{f}}+E^{\mathrm{e}}}{2}=G_{\mathrm{v}}+B_{\mathrm{v}}[J(J+1)-1]-D_{\mathrm{v}}[J(J+1)-1]^{2} \\
E^{\mathrm{f}}-E^{\mathrm{e}}=q_{\mathrm{v}} J(J+1)+q_{\mathrm{D}}[J(J+1)]^{2}
\end{gathered}
$$

Results of the fits are given in Table 5. Six different values of $J_{\max }$ were considered. Whereas $G_{010}$ and $B_{010}$ are almost independent of $J_{\max }$, the corresponding centrifugal distortion constant and the l-type doubling constants $\left(q_{\mathrm{v}}\right.$ and $\left.q_{\mathrm{D}}\right)$ show significant variation which may be indicative of Coriolis coupling with other states. In particular, the $q_{\mathrm{D}}$ value of the $\left(0,1^{1}, 0\right)$ state as calculated with $J_{\max }=$ 110 is only about half as large as the value obtained with $J_{\max }=10$. The $l$-type doubling constant $q_{\mathrm{v}}$ has a negative sign, meaning that the e levels are shifted upward by Coriolis interaction, as expected for the upper state of the interacting pair $\left(0,0^{0}, 1\right) /\left(0,1^{1}, 0\right)$.

For the $\left(1,1^{1}, 0\right)$ state, the situation is quite different compared with the $\left(0,1^{1}, 0\right)$ state, all spectroscopic constants being rather insensitive with respect to variation of $J_{\max }$ up to $J_{\max }=$ 90. As is obvious from the shape of the graph shown in Fig. 4, both $l$-type doubling constants $\left(q_{\mathrm{v}}\right.$ and $\left.q_{\mathrm{D}}\right)$ have the same sign. Predictions for the $\left(0,1^{1}, 0\right)$ and $\left(1,1^{1}, 0\right)$ states of ${ }^{37} \mathrm{ClH}^{35} \mathrm{Cl}^{-}$and ${ }^{37} \mathrm{ClH}^{37} \mathrm{Cl}^{-}$are provided in the ESI $\dagger$ (Table S5).

\subsection{Analysis of Coriolis interaction between low-lying vibrational states of ${ }^{35} \mathrm{ClH}^{35} \mathrm{Cl}^{-}$}

In order to analyse the effects of Coriolis interaction between different vibrational states, we have compared the results of rovibrational calculations with and without inclusion of $\hat{H}_{\text {cor }}$ in the rovibrational Hamiltonian. For the vibrational ground state, the differences in rovibrational term energies (termed $\left.\Delta E_{\text {cor }}(J)\right)$ are extremely small and do not exceed $0.015 \mathrm{~cm}^{-1}$ for $J$ values smaller than 110. Consequently, the effect of neglecting $\hat{H}_{\text {cor }}$ on the rotational and centrifugal distortion constants of this state is negligibly small. For the $\left(0,0^{0}, 1\right)$ states of ${ }^{35} \mathrm{ClH}^{35} \mathrm{Cl}^{-}$ and of ${ }^{37} \mathrm{ClH}^{35} \mathrm{Cl}^{-}$, the situation is quite different (see Fig. 5).

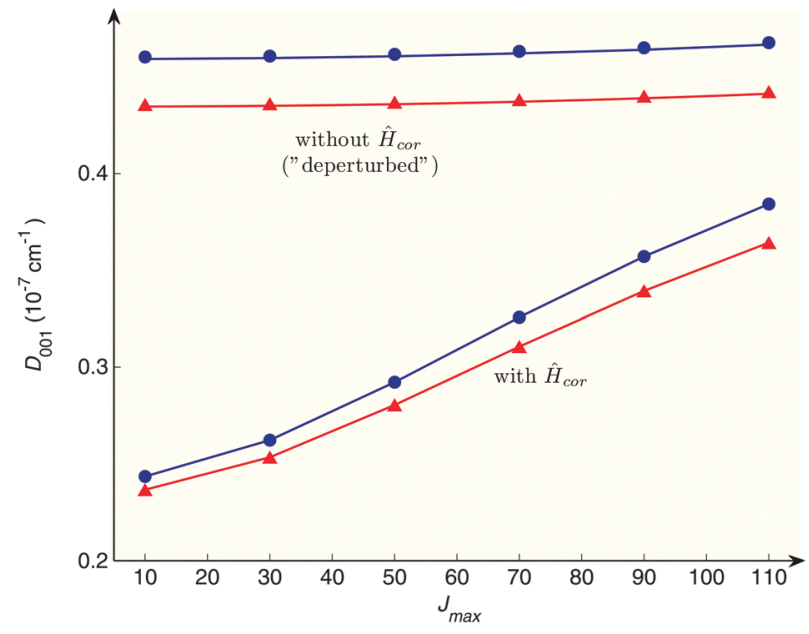

Fig. 5 Dependence of effective and "deperturbed" quartic centrifugal distortion constants on $J_{\max }$ for ${ }^{35} \mathrm{ClH}^{35} \mathrm{Cl}^{-}$(filled circles) and ${ }^{37} \mathrm{ClH}^{35} \mathrm{Cl}^{-}$(triangles).

Neglecting $\hat{H}_{\text {cor }}$, all rovibrational states up to $J_{\max }=110$ are fitted very well without inclusion of the $H_{001}$ term, and the resulting deperturbed $D_{001}$ values vary only slightly between $0.4591 \times$ $10^{-7}$ and $0.4665 \times 10^{-7} \mathrm{~cm}^{-1}$ for ${ }^{35} \mathrm{ClH}^{35} \mathrm{Cl}^{-}$and between $0.4345 \times 10^{-7}$ and $0.4411 \times 10^{-7} \mathrm{~cm}^{-1}$ for ${ }^{37} \mathrm{ClH}^{35} \mathrm{Cl}^{-}$. The values obtained for the former isotopologue agree nicely with the experimental result of $0.4528(61) \times 10^{-7} \mathrm{~cm}^{-1}$ as obtained from Kawaguchi's earlier analysis. ${ }^{4}$ Good agreement is also observed with the earlier theoretical values of Śpirko et al. ${ }^{8}\left(0.42 \times 10^{-7}\right.$ and $0.46 \times 10^{-7} \mathrm{~cm}^{-1}$ from PES I and II, respectively). As is already known from the results of Table 3 , the effective $D_{001}$ values displayed in the lower part of Fig. 5 increase strongly with $J_{\text {max }}$. For the $\left(1,0^{0}, 1\right)$ states of ${ }^{35} \mathrm{ClH}^{35} \mathrm{Cl}^{-}$and of ${ }^{37} \mathrm{ClH}^{35} \mathrm{Cl}^{-}$, the ratios between effective and deperturbed quartic centrifugal distortion constants depend only slightly on $J_{\max }$, varying in the small range between 0.901 and 0.917 . Coriolis interaction thus lowers the $D_{101}$ values by less than $10 \%$.

The graphs of $\Delta E_{\text {cor }}(J)$ for the stretching vibrational states $\left(0,0^{0}, 1\right)$ and $\left(1,0^{\circ}, 1\right)$ are displayed in Fig. 6 (filled black circles).

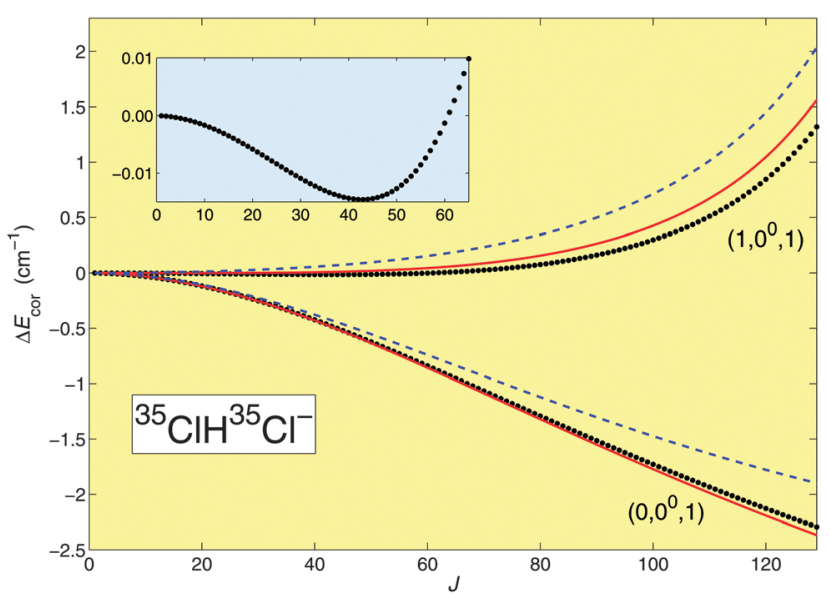

Fig. 6 Contribution of Coriolis interaction to the rovibrational energies of states $\left(0,0^{0}, 1\right)$ and $\left(1,0^{0}, 1\right)$ for ${ }^{35} \mathrm{ClH}^{35} \mathrm{Cl}^{-}$(for details see the text). 
Not surprisingly, and mainly as a result of Coriolis interaction with the higher-lying state $\left(0,1^{1}, 0\right)$, the graph of function $\Delta E_{\text {cor }}(J)$ for the $\left(0,0^{0}, 1\right)$ state has a monotonically decreasing shape. For the $\left(1,0^{0}, 1\right)$ state, the situation is rather different. Over the wide range up to about $J=60$, its graph is almost a horizontal line, with tiny negative values, and then rises rather steeply. In order to rationalize that behaviour and to get more insight into the relevant Coriolis interaction, we have set up a number of model calculations involving a small number of interacting vibrational states. In an obvious shorthand notation including energetic numbering in ascending order (in parentheses), these are 001(1), 010(2), 101(3), 110(4), and 210(5). Direct Coriolis interaction of states $\left(0,0^{\circ}, 1\right)$ and $\left(1,0^{\circ}, 1\right)$ occurs with states of type $\left(v_{1}, 1^{1}, 0\right)$. For a given value of $J$, the chosen model Hamiltonian has the following form:

$$
\begin{aligned}
H_{\text {model }}(J)= & \left(\begin{array}{lllll}
E_{001}(J) & & \multicolumn{3}{c}{\text { sym. }} \\
\epsilon_{21} X & E_{010}(J) & & \\
0 & \epsilon_{32} X & E_{101}(J) & \\
\epsilon_{41} X & 0 & \epsilon_{43} X & E_{110}(J) & \\
\epsilon_{51} X & 0 & \epsilon_{53} X & 0 & E_{210}(J)
\end{array}\right) \\
& \text { with } X=\sqrt{J(J+1)}
\end{aligned}
$$

The $J$-dependent diagonal elements of the model Hamiltonian are obtained as eigenvalues of $\hat{H}-\hat{H}_{\text {cor }}$ (see eqn (5)), whereas the non-vanishing off-diagonal elements are chosen of the form $\epsilon_{i j} \sqrt{J(J+1)}$. Here, the parameters $\epsilon_{i j}$ are calculated as matrix elements of $\hat{H}_{\text {cor }}$ over the rovibrational eigenfunctions of $\hat{H}-\hat{H}_{\text {cor }}$ for $J=1$. According to the present calculations, the matrix elements of $\hat{H}-\hat{H}_{\text {cor }}$ are almost independent of $J$ and thus the use of constant values is justified well. E.g., we compute $\epsilon_{21}(J=1)=0.1376 \mathrm{~cm}^{-1}$ and $\epsilon_{21}(J=120)=$ $0.1292 \mathrm{~cm}^{-1}$. For ${ }^{35} \mathrm{ClH}^{35} \mathrm{Cl}^{-}$, the numerical values chosen for the interaction parameters are (in $\mathrm{cm}^{-1}$ ): $\epsilon_{21}=0.1376$, $\epsilon_{41}=0.1021, \epsilon_{51}=-0.0485, \epsilon_{32}=-0.1066, \epsilon_{43}=0.0589$, and $\epsilon_{53}=-0.1101$.

Results of the model calculations as obtained by diagonalizing $H_{\text {model }}$ for different values of $J$ are included in Fig. 6. The functions $\Delta E_{\text {cor }}(J)$ are defined only pointwise, but for better visibility we have partly chosen them to be continuous functions. Very good agreement with the variational calculations is obtained by the full model with five states and six non-vanishing offdiagonal matrix elements (full red lines). A reduced two-state model for the proton stretching vibrational state, involving states 001 and 010 (dashed blue line in lower part of Fig. 6), works reasonably well for low values of $J$, but deviates significantly from the variational results for $J>50$.

The upper state $\left(1,0^{0}, 1\right)$ considered in Fig. 6 is shifted upward through direct Coriolis interaction with state 010 and downward through interaction with state 110. A minimum of these three states (see dashed blue line in the upper part of Fig. 6) is thus required to obtain a qualitatively correct form of $\Delta E_{\text {cor }}(J)$. Owing to the rather large value of $\epsilon_{53}$, interaction with state 210 is also significant.

The model Hamiltonian was also employed to study the $J$ dependence of the $\mathrm{f}-\mathrm{e}$ splitting in the $\left(0,1^{1}, 0\right)$ state of ${ }^{35} \mathrm{ClH}^{35} \mathrm{Cl}^{-}$(see ESI $\dagger$ Fig. S1). A two-state model involving states 010 and 001 is clearly inadequate to properly describe the $J$ dependence of that splitting for $J>20$. The situation is much improved by inclusion of state 101, and almost quantitative agreement with the full variational calculations is obtained for a four-state model in which state 201 is added to the threestate model.

\subsection{Predictions for deuterated isotopologues}

High-resolution IR studies for deuterated isotopologues of the hydrogen bichloride ion are not yet available and so theory may provide useful predictions. For this purpose, we have carried out variational calculations of rovibrational states and transition dipole moments among them on the basis of the analytical PEFs and EDMFs discussed earlier, the latter being modified appropriately to account for the change in nuclidic masses. The most important results are presented in a compact manner in Table 6, which comprises spectroscopic constants for the vibrational ground state, the three singly-excited vibrational states and the doubly excited states $\left(1,0^{0}, 1\right)$ and $\left(1,1^{1} 0\right)$. In the case of the states with $l=1$, the $l$-type doubling constants $q_{\mathrm{v}}$ and $q_{\mathrm{D}}$ are quoted as well. Details of the fitting procedures employed to determine the spectroscopic constants are given in the footnotes to the table.

Upon deuterium substitution, the symmetric stretching vibration $\nu_{1}$ experiences a slight increase in wavenumber, ranging between $1.76 \mathrm{~cm}^{-1}$ for ${ }^{35} \mathrm{ClD}^{35} \mathrm{Cl}^{-}$and $1.82 \mathrm{~cm}^{-1}$ for ${ }^{37} \mathrm{ClD}^{37} \mathrm{Cl}^{-}$. Such an increase is not allowed within the harmonic approximation and must be the result of the anharmonic nature of the potential energy surface. The origins of the $\nu_{3}$ bands of the deuterated species ${ }^{35} \mathrm{ClD}^{35} \mathrm{Cl}^{-}$and ${ }^{37} \mathrm{ClD}^{35} \mathrm{Cl}^{-}$, and ${ }^{37} \mathrm{ClD}^{37} \mathrm{Cl}^{-}$ are predicted at $483.92,483.88$, and $483.84 \mathrm{~cm}^{-1}$, with an estimated accuracy of better than $1 \mathrm{~cm}^{-1}$. In contrast to the $\mathrm{H}$ isotopologues, no unusual effects are predicted upon ${ }^{37} \mathrm{Cl}$ substitution. A neon matrix-isolation IR spectroscopic study by Forney et $a .^{20}$ reported absorptions at 489.3 and $496.2 \mathrm{~cm}^{-1}$, both of which were attributed to $\mathrm{ClDCl}^{-}$. Like for the most abundant isotopologue, the neon matrix environment produces a slight blue shift in the antisymmetric stretching vibration. The earlier argon-matrix studies ${ }^{21}$ yielded $\nu_{3}\left(\mathrm{ClDCl}^{-}\right)=$ $463 \mathrm{~cm}^{-1}$ and thus a red-shift of $21 \mathrm{~cm}^{-1}$ with respect to the present prediction. While no neon-matrix value is available for the combination tone $\nu_{1}+\nu_{3}\left(\mathrm{ClDCl}^{-}\right)$, a weak, relatively broad absorption at $730 \mathrm{~cm}^{-1}$ observed in an argon matrix was assigned to that band. ${ }^{21}$ It also exhibits a red-shift of $21 \mathrm{~cm}^{-1}$ with respect to our theoretical value (Table 6).

The deuteron bending vibrational energy of ${ }^{35} \mathrm{ClD}^{35} \mathrm{Cl}^{-}$is predicted to be $G_{010}=571.32 \mathrm{~cm}^{-1}$, with an uncertainty of $c a .1 \mathrm{~cm}^{-1}$. Again, the energy is higher than that of the deuteron stretching vibration $\left(G_{001}=483.92 \mathrm{~cm}^{-1}\right)$, the difference of $87.40 \mathrm{~cm}^{-1}$ being even larger than in the case of ${ }^{35} \mathrm{ClH}^{35} \mathrm{Cl}^{-}\left(72.85 \mathrm{~cm}^{-1}\right)$. Consequently, the $l$-type doubling constant $q_{\mathrm{v}}$ again has a negative value. 
Table 6 Predicted effective spectroscopic constants (in $\mathrm{cm}^{-1}$ ) for deuterated isotopologues $^{a}$

\begin{tabular}{lllllll}
\hline Isotopologue & State & $G_{\mathrm{v}}$ & $B_{\mathrm{v}}$ & $D_{\mathrm{v}}$ & $q_{\mathrm{v}}\left(\times 10^{3}\right)$ & $q_{D}\left(\times 10^{7}\right)$ \\
${ }^{35} \mathrm{ClD}^{35} \mathrm{Cl}^{-}$ & $\left(0,0^{0}, 0\right)$ & $989.49^{b}$ & 0.097724 & 0.3940 & & \\
& $\left(1,0^{0}, 0\right)$ & 304.52 & 0.097056 & 0.4389 & & \\
& $\left(0,0^{0}, 1\right)^{c}$ & 483.92 & 0.093706 & 0.2934 & & \\
& $\left(0,1^{1}, 0\right)$ & 571.32 & 0.098330 & 0.4988 & -0.231 & 0.16 \\
& $\left(1,0^{0}, 1\right)$ & 750.77 & 0.093076 & 0.4424 & & \\
& $\left(1,1^{1}, 0\right)$ & 868.74 & 0.097467 & 0.5163 & -0.018 & 0.07 \\
${ }^{37} \mathrm{ClD}^{35} \mathrm{Cl}^{-}$ & $\left(0,0^{0}, 0\right)$ & $986.98^{b}$ & 0.095086 & 0.3728 & & \\
& $\left(1,0^{0}, 0\right)$ & 300.53 & 0.094447 & 0.4144 & & \\
& $\left(0,0^{0}, 1\right)^{d}$ & 483.88 & 0.091187 & 0.2821 & & \\
& $\left(0,1^{1}, 0\right)$ & 571.13 & 0.095671 & 0.4701 & -0.216 & 0.15 \\
& $\left(1,0^{0}, 1\right)$ & 747.27 & 0.090583 & 0.4190 & & \\
& $\left(1,1^{1}, 0\right)$ & 864.69 & 0.094847 & 0.4868 & -0.014 & 0.07 \\
${ }^{37} \mathrm{ClD}^{37} \mathrm{Cl}^{-}$ & $\left(0,0^{0}, 0\right)$ & $984.45^{b}$ & 0.092452 & 0.3523 & & \\
& $\left(1,0^{0}, 0\right)$ & 296.48 & 0.091842 & 0.3906 & & \\
& $\left(0,0^{0}, 1\right)^{e}$ & 483.84 & 0.088671 & 0.2707 & & \\
& $\left(0,1^{1}, 0\right)$ & 570.95 & 0.093016 & 0.4424 & -0.202 & 0.14 \\
& $\left(1,0^{0}, 1\right)$ & 743.73 & 0.088093 & 0.3963 & & \\
& $\left(1,1^{1}, 0\right)$ & 860.59 & 0.092231 & 0.4581 & -0.010 & 0.06
\end{tabular}

${ }^{a}$ Obtained by least-squares fit to rovibrational energies up to $J_{\max }=30$. ${ }^{b}$ Zero-point energy. ${ }^{c} H_{001}=-0.819 \times 10^{-12} \mathrm{~cm}^{-1} \cdot{ }^{d} H_{001}=-0.733 \times$ $10^{-12} \mathrm{~cm}^{-1} \cdot{ }^{e} H_{001}=-0.654 \times 10^{-12} \mathrm{~cm}^{-1}$.

As was already found in the calculations by Špirko et al., deuterium substitution leads to an increase in the rotational constant $B_{000}$. For ${ }^{35} \mathrm{ClD}^{35} \mathrm{Cl}^{-}$, we calculate $\Delta B_{000}=$ $0.000357 \mathrm{~cm}^{-1}$, in excellent agreement with the previous prediction of $0.00037 \mathrm{~cm}^{-1}$. $^{8}$ The increase is still more pronounced for $B_{001}$ and $B_{101}$, where differences of 0.001424 and $0.001271 \mathrm{~cm}^{-1}$ are obtained. On the other hand, $B_{010}$ $\left({ }^{35} \mathrm{ClD}^{35} \mathrm{Cl}^{-}\right)$exceeds $B_{010}\left({ }^{35} \mathrm{ClH}^{35} \mathrm{Cl}^{-}\right)$by only $0.000197 \mathrm{~cm}^{-1}$, smaller than the difference for the vibrational ground state.

\subsection{Transition dipole moments}

Squares of transition dipole moments for two series of vibrational transitions of ${ }^{35} \mathrm{ClH}^{35} \mathrm{Cl}^{-}$and ${ }^{35} \mathrm{ClD}^{35} \mathrm{Cl}^{-}$are listed in Table 7. The first series arises from the vibrational ground state, while the second one corresponds to hot transitions starting from the first excited state of the symmetric stretching vibration with energies of $303 \mathrm{~cm}^{-1}\left({ }^{35} \mathrm{ClH}^{35} \mathrm{Cl}^{-}\right)$and $305 \mathrm{~cm}^{-1}$ $\left({ }^{35} \mathrm{ClD}^{35} \mathrm{Cl}^{-}\right)$above the vibrational ground-state. As expected, the largest $\mu^{2}$ values are obtained for the $\nu_{3}$ bands. The present values of $2.011 \mathrm{D}^{2}$ and $1.942 \mathrm{D}^{2}$ are only slightly larger than the results of the earlier CEPA calculations $\left(1.777\right.$ and $\left.1.793 \mathrm{D}^{2}\right){ }^{5}$ Among the transitions considered in Table 7, only two of them are allowed within the familiar double-harmonic approximation (DHA). These are the fundamental transition $\left(0,0^{0}, 1\right) \leftarrow\left(0,0^{0}, 0\right)$ and the hot band $\left(1,0^{0}, 1\right) \leftarrow\left(1,0^{\circ}, 0\right)$. Actually, it is mechanical anharmonicity arising from the strongly anharmonic nature of the PES, consideration of which is decisive to arrive at accurate values for the transition dipole moments. Including only the linear EDMF term $D_{001}^{\prime \prime}$ (see ESI $\dagger$ Table S2) in the calculations, $\mu^{2}$ values for the two transitions are obtained, which differ from those of the full treatment by less than 13\%. Interestingly, it is not the DHA-allowed
Table 7 Squares of transition dipole moments $\mu_{\mathrm{vv}^{\prime}}{ }^{2}$ (in $\mathrm{D}^{2}$ ) for stretching vibrational transitions of ${ }^{35} \mathrm{ClH}^{35} \mathrm{Cl}^{-}$and ${ }^{35} \mathrm{CID}^{35} \mathrm{Cl}^{-}$

\begin{tabular}{|c|c|c|c|c|c|}
\hline \multirow[b]{2}{*}{ Upper state } & \multirow[b]{2}{*}{ Lower state } & \multicolumn{2}{|c|}{${ }^{35} \mathrm{ClH}^{35} \mathrm{Cl}^{-}$} & \multicolumn{2}{|c|}{${ }^{35} \mathrm{ClD}^{35} \mathrm{Cl}^{-}$} \\
\hline & & Full $^{a}$ & 1-Term ${ }^{b}$ & Full $^{a}$ & 1 -Term ${ }^{b}$ \\
\hline$\left(0,0^{0}, 1\right)$ & $\left(0,0^{0}, 0\right)$ & 2.011 & 2.262 & 1.942 & 2.132 \\
\hline$\left(1,0^{0}, 1\right)$ & & 0.664 & 0.731 & 0.324 & 0.344 \\
\hline$\left(2,0^{0}, 1\right)$ & & 0.145 & 0.158 & 0.044 & 0.047 \\
\hline$\left(3,0^{0}, 1\right)$ & & 0.026 & 0.028 & 0.005 & 0.005 \\
\hline$\left(0,0^{0}, 1\right)$ & $\left(1,0^{0}, 0\right)$ & 1.488 & 1.707 & 1.087 & 1.222 \\
\hline$\left(1,0^{0}, 1\right)$ & & 0.603 & 0.692 & 1.147 & 1.283 \\
\hline$\left(2,0^{0}, 1\right)$ & & 0.760 & 0.840 & 0.485 & 0.514 \\
\hline$\left(3,0^{0}, 1\right)$ & & 0.313 & 0.342 & 0.116 & 0.123 \\
\hline
\end{tabular}

${ }^{a}$ Complete EDMF (see ESI Table S2) is employed. ${ }^{b}$ Only the EDMF term $D_{001}^{\prime \prime}$ is employed.

transition which has the largest $\mu^{2}$ value of the hot bands of ${ }^{35} \mathrm{ClH}^{35} \mathrm{Cl}^{-}$. Instead, a value larger by a factor of 2.5 is calculated for the DHA-forbidden transition $\left(0,0^{0}, 1\right) \leftarrow\left(1,0^{0}, 0\right)$. The situation is different for ${ }^{35} \mathrm{ClD}^{35} \mathrm{Cl}^{-}$, where $\mu^{2}$ for the DHA-allowed transition $\left(1.147 \mathrm{D}^{2}\right)$ is slightly larger than for the noted DHA-forbidden transition. However, again owing to high mechanical anharmonicity, the $\mu^{2}$ value for the DHA-allowed hot transition in ${ }^{35} \mathrm{ClD}^{35} \mathrm{Cl}^{-}$ exceeds the corresponding value for ${ }^{35} \mathrm{ClH}^{35} \mathrm{Cl}^{-}$by a factor of 1.9 , which must be considered a rather unusual deuterium effect.

In contrast to $\mathrm{FHF}^{-}$and $\mathrm{FDF}^{-}$, where 14 and 22 lines were observed in the $\nu_{2}$ bands, ${ }^{2,3}$ and relatively large $\mu^{2}$ values of 0.051 and $0.042 \mathrm{D}^{2}$ were calculated recently, ${ }^{42}$ a much smaller value of $0.0050 \mathrm{D}^{2}$ was computed for the $\nu_{2}$ band of ${ }^{35} \mathrm{ClH}^{35} \mathrm{Cl}^{-}$. Since Coriolis interaction between the $\left(0,0^{0}, 1\right)$ and $\left(0,1^{1}, 0\right)$ states of ${ }^{35} \mathrm{ClH}^{35} \mathrm{Cl}^{-}$is much weaker, intensity borrowing is less pronounced for the bichloride ion.

\section{Conclusions}

High-resolution spectroscopic investigations of hydrogenbonded anionic complexes are very scarce and essentially limited to the pioneering work of Kawaguchi and Hirota on $\mathrm{FHF}^{-}$and $\mathrm{ClHCl}^{-1-4}$ For the less strongly bound species ${ }^{35} \mathrm{ClH}^{35} \mathrm{Cl}^{-}$and ${ }^{37} \mathrm{ClH}^{35} \mathrm{Cl}^{-}$, only the $\nu_{3}$ bands (corresponding to the proton stretching vibrations) were analysed, exhibiting indication of Coriolis interaction with the higher-lying proton bending vibrational state. ${ }^{4}$ With the aid of accompanying highlevel quantum chemical calculations, 45 and 41 lines within the $\nu_{1}+\nu_{3}$ combination tones of ${ }^{35} \mathrm{ClH}^{35} \mathrm{Cl}^{-}$and ${ }^{37} \mathrm{ClH}^{35} \mathrm{Cl}^{-}$ observed earlier could now be faithfully assigned. The variational calculations of rovibrational energies and wave functions are based on Watson's Hamiltonian for linear molecules ${ }^{34}$ and an empirically corrected $\operatorname{CCSD}\left(\mathrm{T}^{*}\right)-\mathrm{F} 12 \mathrm{~b}$ potential energy surface. In contrast to the earlier work on the $\nu_{3}$ bands, ${ }^{4}$ all lines could be fitted very well with just three parameters $\left(G_{\mathrm{v}}, B_{\mathrm{v}}\right.$, and $\left.D_{\mathrm{v}}\right)$. However, that does not mean that Coriolis interaction plays no role for the $\left(1,0^{0}, 1\right)$ vibrational state. Instead, computations with a five-state model Hamiltonian show that more than three interacting states are required to quantitatively describe the variation of the Coriolis contribution to the rovibrational energies within state $\left(1,0^{0}, 1\right)$ up to high values of $J$. 


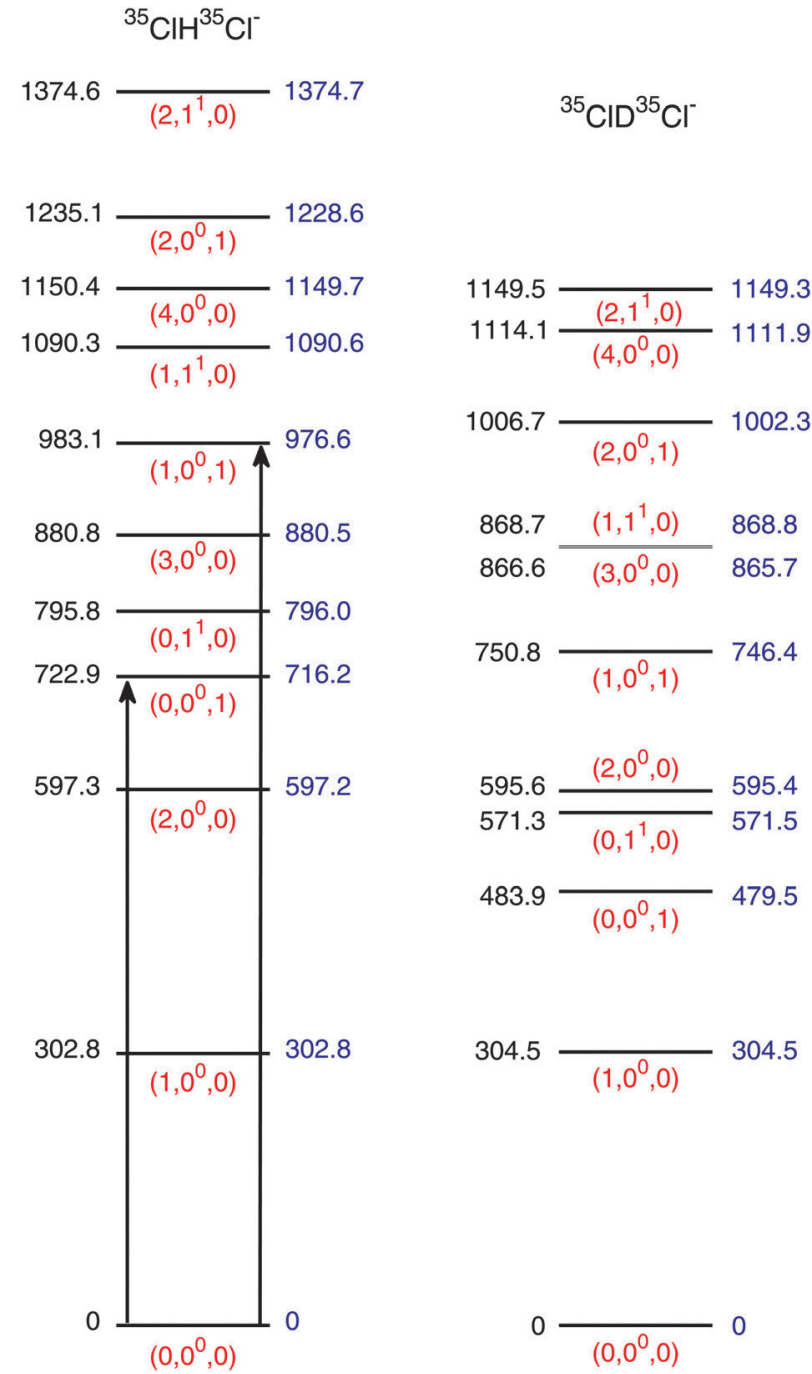

Fig. 7 Calculated term energies (in $\mathrm{cm}^{-1}$ ) for lowest 11 vibrational states of ${ }^{35} \mathrm{ClH}^{35} \mathrm{Cl}^{-}$and ${ }^{35} \mathrm{CID}^{35} \mathrm{Cl}^{-}$. Left columns: data obtained with empirically corrected PEF; right columns: data obtained with $\operatorname{CCSD}\left(T^{\star}\right)-F 12 \mathrm{~b} / \mathrm{AV} 5 Z$ PEF Observed transitions are indicated by arrows.

The $\nu_{3}$ and $\nu_{1}+\nu_{3}$ bands of $\mathrm{ClHCl}^{-}$isotopologues are characterized by band heads which are a rare issue in rovibrational spectroscopy. For ${ }^{35} \mathrm{ClH}^{35} \mathrm{Cl}^{-}$and ${ }^{37} \mathrm{ClH}^{35} \mathrm{Cl}^{-}$the heads of the $\nu_{3}$ bands are calculated at line $\mathrm{R}(18)$ with wavenumbers of 724.665 and $724.681 \mathrm{~cm}^{-1}$, respectively. For the combination tone, we predict the band heads to occur at $\mathrm{R}(16)$ with wavenumbers of 984.729 and $981.333 \mathrm{~cm}^{-1}$.

According to the present calculations, the Coriolis interaction between states $\left(0,0^{0}, 1\right)$ and $\left(0,1^{1}, 0\right)$ is relatively weak such that no significant intensity borrowing takes place and no line within the $\nu_{2}$ band could be observed so far. Our calculations predict $G_{010}=795.75 \mathrm{~cm}^{-1}$, with an uncertainty of $c a .1 \mathrm{~cm}^{-1}$. We thus support Kawaguchi's earlier estimate of $792 \pm 9 \mathrm{~cm}^{-1},{ }^{4}$ which was based on a two-state model and an educated guess of the Coriolis coupling constant $\eta$.

Finally, Fig. 7 presents an overview of the 11 vibrational states of ${ }^{35} \mathrm{ClH}^{35} \mathrm{Cl}^{-}$and ${ }^{35} \mathrm{ClD}^{35} \mathrm{Cl}^{-}$which we have investigated in the present work, including their calculated vibrational energies. The two arrows indicate the transitions which have been observed by Kawaguchi. ${ }^{4}$ The figure includes data from calculations with both empirically corrected and uncorrected PEFs. The latter differ from experiment by $6.7 \mathrm{~cm}^{-1}$ for both $\nu_{3}$ and $\nu_{1}+\nu_{3}$. Most of the error in the $\operatorname{CCSD}\left(\mathrm{T}^{*}\right)-\mathrm{F} 12 \mathrm{~b}$ calculations probably results from the neglect of core valence $(\mathrm{CV})$ correlation and scalar relativistic effects. We have investigated the former by means of standard $\operatorname{CCSD}(T)^{10}$ in conjunction with the ACVQZ basis set, whereas the latter were studied within the Douglas-Kroll-Hess (DKH) approximation of 2 nd order ${ }^{43,44}$ using the AVTZ basis set. Inclusion of CV correlation increases $\nu_{3}$ by $4.5 \mathrm{~cm}^{-1}$ and the DKH correction amounts to $+4.7 \mathrm{~cm}^{-1}$. Adding the sum to the uncorrected $\nu_{3}$ value, we arrive at $725.8 \mathrm{~cm}^{-1}$ and thus at an overestimate by $2.9 \mathrm{~cm}^{-1}$. The latter difference with respect to experiment is attributed to the neglect of higher-order correlation effects (in particular quadruple substitutions) and potentially Born-Oppenheimer breakdown contributions. The explicit consideration of higherorder correlation contributions would be very expensive for $\mathrm{ClHCl}^{-}$, since time-consuming calculations at many different nuclear configurations would have to be carried out.

The information of Fig. 7 may be helpful to forthcoming spectroscopic work on the bichloride system. In particular, assignments of lines within the hot band $\left(1,0^{\circ}, 1\right) \leftarrow\left(1,0^{0}, 0\right)$ would be of great interest since it would open the way to a precise determination of the $\nu_{1}$ band origin. Unfortunately, the $\mu^{2}$ value for that band is relatively low (see Table 7) and the situation appears to be less favorable than for $\mathrm{FHF}^{-}$where the corresponding transition was observed earlier. ${ }^{2}$ Extension of the previous spectroscopic work to a transition to the first overtone of the proton stretching vibration $\left(0,0^{0}, 2\right)$ appears to be of interest as well. For ${ }^{35} \mathrm{ClH}^{35} \mathrm{Cl}^{-}$, its vibrational term value is predicted at $1746.3 \mathrm{~cm}^{-1}$, with effective rotational and quartic centrifugal distortion constants of $0.09339 \mathrm{~cm}^{-1}$ and $0.270 \times 10^{-7} \mathrm{~cm}^{-1}$ from fits with $J_{\max }=70$, respectively. According to our calculations, observation might be possible in the form of a hot band with initial state $\left(0,0^{\circ}, 1\right)$, the band origin being predicted at $1023.4 \mathrm{~cm}^{-1}$ and the transition dipole moment calculated to be $1.429 \mathrm{D}$.

\section{References}

1 K. Kawaguchi and E. Hirota, J. Chem. Phys., 1986, 84, 2953.

2 K. Kawaguchi and E. Hirota, J. Chem. Phys., 1987, 87, 6838.

3 K. Kawaguchi and E. Hirota, J. Mol. Struct., 1995, 352/ 353, 389.

4 K. Kawaguchi, J. Chem. Phys., 1988, 88, 4186.

5 P. Botschwina, P. Sebald and R. Burmeister, J. Chem. Phys., 1988, 88, 5246.

6 W. Meyer, J. Chem. Phys., 1973, 58, 1017.

7 S. Ikuta, T. Saitoh and O. Nomura, J. Chem. Phys., 1989, 91, 3539.

8 V. Špirko, A. Čejchan and G. H. F. Diercksen, Chem. Phys., 1991, 151, 45. 
9 J. E. Del Bene and M. J. T. Jordan, Spectrochim. Acta, Part A, 1999, 55, 719.

10 K. Raghavachari, G. W. Trucks, J. A. Pople and M. Head-Gordon, Chem. Phys. Lett., 1989, 157, 479.

11 T. H. Dunning, Jr., J. Chem. Phys., 1989, 90, 1007.

12 R. A. Kendall, T. H. Dunning, Jr. and R. J. Harrison, J. Chem. Phys., 1992, 96, 6796.

13 D. E. Woon and T. H. Dunning, Jr., J. Chem. Phys., 1993, 98, 1358.

14 P. Sebald, Chem. Phys., 2008, 346, 77.

15 H.-J. Werner, T. B. Adler, G. Knizia and F. R. Manby, in Recent Progress in Coupled Cluster Methods: Theory and Applications, ed. P. Carsky, J. Pitter and J. Paldus, Springer, 2010.

16 D. P. Tew, C. Hättig, R. A. Bachorz and W. Klopper, in Recent Progress in Coupled Cluster Methods: Theory and Applications, ed. P. Carsky, J. Pitter and J. Paldus, Springer, 2010.

17 C. Hättig, W. Klopper, A. Köhn and D. P. Tew, Chem. Rev., 2012, 112, 4.

18 L. Kong, F. A. Bischoff and E. F. Valeev, Chem. Rev., 2012, 112, 75 .

19 K. Kawaguchi, C. Yamada, S. Saito and E. Hirota, J. Chem. Phys., 1985, 82, 1750.

20 D. Forney, M. E. Jacox and W. E. Thompson, J. Chem. Phys., 1995, 103, 1755.

21 D. E. Milligan and M. E. Jacox, J. Chem. Phys., 1970, 53, 2034.

22 T. B. Adler, G. Knizia and H.-J. Werner, J. Chem. Phys., 2007, 127, 221106.

23 G. Knizia, T. B. Adler and H.-J. Werner, J. Chem. Phys., 2009, 130, 054104.

24 H.-J. Werner, G. Knizia, T. B. Adler and O. Marchetti, Z. Phys. Chem., 2010, 224, 493.

25 T. H. Dunning, Jr., K. A. Peterson and A. K. Wilson, J. Chem. Phys., 2001, 114, 9244 and references therein.
26 K. E. Yousaf and K. A. Peterson, Chem. Phys. Lett., 2009, 476, 303.

27 F. Weigend, Phys. Chem. Chem. Phys., 2002, 4, 4285.

28 C. Hättig, Phys. Chem. Chem. Phys., 2005, 7, 59.

29 K. A. Peterson, T. B. Adler and H.-J. Werner, J. Chem. Phys., 2008, 128, 084102.

30 H.-J. Werner, P. J. Knowles, R. Lindh, F. R. Manby and M. Schütz, et al., MOLPRO version 2009.1, a package of $a b$ initio programs, 2009, see http://www.molpro.net.

31 P. Botschwina, P. Sebald, D. Figgen and H. Stoll, Mol. Phys., 2007, 105, 1193.

32 P. Sebald, R. Oswald, P. Botschwina, H. Stoll and D. Figgen, J. Phys. Chem. A, 2009, 113, 11772.

33 P. Sebald, H. Vennekate, R. Oswald, P. Botschwina and H. Stoll, Mol. Phys., 2010, 108, 487.

34 J. K. G. Watson, Mol. Phys., 1970, 19, 465.

35 R. J. Whitehead and N. C. Handy, J. Mol. Spectrosc., 1975, 55, 356.

36 R. N. Zare, Angular Momentum, John Wiley \& Sons Inc, New York, 1988.

37 S. Carter, J. Senekowitsch, N. C. Handy and P. Rosmus, Mol. Phys., 1987, 65, 143.

38 R. Herman and R. F. Wallis, J. Chem. Phys., 1955, 23, 637.

39 J. K. G. Watson, J. Mol. Spectrosc., 1987, 125, 428.

40 J. K. G. Watson, Can. J. Phys., 2001, 79, 521 and references therein.

41 J. M. Brown, J. T. Hougen, K.-P. Huber, J. W. C. Johns, I. Kopp, H. Lefebvre-Brion, A. J. Merer, D. A. Ramsey, J. Rostas and R. N. Zare, J. Mol. Spectrosc., 1975, 55, 500.

42 P. Sebald, A. Bargholz, R. Oswald, C. Stein and P. Botschwina, J. Phys. Chem. A, DOI: 10.1021/jp3123677.

43 M. Douglas and N. M. Kroll, Ann. Phys., 1974, 82, 89.

44 G. Jansen and B. A. Hess, Phys. Rev. A: At., Mol., Opt. Phys., 1989, 39, 6016. 